\title{
Opportunities for Biomanufacturing in Low Earth Orbit: Current Status and Future Directions
}

Marc A. Giulianotti ${ }^{1 *}$, Arun Sharma ${ }^{2,3}$, Rachel A. Clemens ${ }^{4}$, Orquidea Garcia ${ }^{5}$, D. Lancing Taylor ${ }^{6}$, Nicole L. Wagner ${ }^{7}$, Kelly A. Shepard ${ }^{8}$, Anjali Gupta ${ }^{4}$, Siobhan Malany ${ }^{9}$, Alan J. Grodzinsky ${ }^{10}$, Mary Kearns-Jonker ${ }^{11}$, Devin B. Mair ${ }^{12}$, Deok-Ho Kim ${ }^{12,13}$, Michael S. Roberts ${ }^{1}$, Jeanne F. Loring ${ }^{14}$, Jianying Hu${ }^{15}$, Lara E. Warren ${ }^{1}$, Sven Eenmaa ${ }^{1}$, Joe Bozada ${ }^{16}$, Eric Paljug ${ }^{16}$, Mark Roth ${ }^{17}$, Donald P. Taylor ${ }^{18}$, Gary Rodrigue ${ }^{1}$, Patrick Cantini ${ }^{19}$, Amelia W. Smith ${ }^{1}$, William R. Wagner ${ }^{19,20^{*}}$

1 Center for the Advancement of Science in Space, Melbourne, FL, USA

2 Board of Governors Regenerative Medicine Institute, Cedars-Sinai Medical Center, Los

Angeles, CA, USA

3 Smidt Heart Institute, Cedars-Sinai Medical Center, Los Angeles, CA, USA

4 Axiom Space, Inc., Houston, TX, USA

5 Johnson \& Johnson 3D Printing Innovation \& Customer Solutions, Johnson \& Johnson

Services, Inc., Irvine, CA, USA.

6 University of Pittsburgh Drug Discovery Institute and Department of Computational and Systems Biology, University of Pittsburgh, Pittsburgh, PA, USA

7 LambdaVision Inc., Farmington, CT, USA

8 California Institute for Regenerative Medicine, Oakland, California, USA

9 Department of Pharmacodynamics, College of Pharmacy, University of Florida, Gainesville, FL USA

10 Departments of Biological Engineering, Mechanical Engineering and Electrical Engineering and Computer Science, Massachusetts Institute of Technology, Cambridge, MA, USA

11 Department of Pathology and Human Anatomy, Loma Linda University School of Medicine, Loma Linda, CA, USA

12 Department of Biomedical Engineering, Johns Hopkins University School of Medicine,

Baltimore, MD, USA

13 Department of Medicine, Johns Hopkins University School of Medicine, Baltimore, MD, USA

14 Scripps Research Institute, San Diego, CA, USA

15 Center for Computational Health IBM Research, Yorktown Heights, NY, USA

16 Joseph M. Katz Graduate School of Business, University of Pittsburgh, Pittsburgh, PA, USA

17 Pittsburgh, PA, USA

18 The Ohio State University, Columbus, OH, USA

19 McGowan Institute for Regenerative Medicine, Pittsburgh, PA, USA

20 Departments of Surgery, Bioengineering, Chemical Engineering, University of Pittsburgh, Pittsburgh, PA, USA

*Correspondence: mgiulianotti@issnationallab.org and wagnerwr@upmc.edu

Keywords: microgravity, stem cells, microphysiological systems, organoids, biofabrication 


\begin{abstract}
In humankind's endeavor to explore beyond our planet and travel further into space, we are now at the threshold of an era in which it is possible to move to and from low Earth orbit (LEO) with increasing ease and reduced cost. Through the International Space Station (ISS) U.S. National Laboratory, investigators from industry, academia, and government can easily access the unique LEO environment on the ISS to conduct research and development (R\&D) activities in ways not possible on Earth.
\end{abstract}

A key advantage of the LEO environment for life sciences research is the ability to conduct experiments in sustained microgravity conditions. The ability to conduct long-term research in microgravity enables opportunities for novel, fundamental studies in tissue engineering and regenerative medicine, including research on stem cell proliferation and differentiation, biofabrication, and disease modeling using microphysiological systems (MPS) that build on prior research using simulated microgravity conditions (Grimm, D., et al. 2018). Over the last decade, space-based research has demonstrated that microgravity informs our knowledge of fundamental biology and accelerates advancements in health care and medical technologies (International Space Station 2019). The benefits provided by conducting biomedical research in LEO may lead to breakthroughs not achievable on Earth.

We are now at a transition point, in which nations are changing their approach to space-based $R \& D$. The focus is shifting from government-funded fundamental science toward the expansion of privately funded R\&D with terrestrial application and economic value that will drive a robust marketplace for innovation and manufacturing in LEO. Making this long-term transition requires public-private participation and near-term funding to support critical R\&D to leverage the benefits of the LEO environment and de-risk space-based research.

Studies conducted on the ISS over the past several years have indicated that one area with potential significant economic value and benefit to life on Earth is space-based biomanufacturing, or the use of biological and nonbiological materials to produce commercially relevant biomolecules and biomaterials for use in preclinical, clinical, and therapeutic applications. We must take advantage of the remaining lifetime of the ISS as a valuable LEO platform to demonstrate this economic value and Earth benefit. By facilitating access to the space station, the ISS National Lab is uniquely positioned to enable the R\&D necessary to bridge the gap between the initial discovery phase of space-based biomedical research and the development of a sustainable, investment-worthy biomanufacturing market in LEO supported by future commercial platforms.

Through a joint effort, the Center for the Advancement of Science in Space (CASIS), which manages the ISS National Lab, and the University of Pittsburgh's McGowan Institute for Regenerative Medicine brought together thought leaders from around the U.S. for a Biomanufacturing in Space Symposium that consisted of a series of working sessions to review data from past space-based tissue engineering and regenerative medicine research, discuss 
relevant current space-based $R \& D$ in this area, and consider potential future markets to address the questions: What are the most promising opportunities to leverage the ISS to advance space-based biomanufacturing moving forward? What are the current gaps or barriers that, if overcome, could clear pathways toward private investment in LEO as a valued site for research, development, and production activity? And, most importantly: For which opportunities do the most compelling value propositions exist?

The goal of the Biomanufacturing in Space Symposium was to help identify the specific areas in which government and industry investment would be most likely to stimulate advancements that overcome barriers. This would lead to a more investment-ready landscape for private interests to enter the market and fuel exponential growth. The symposium was meant to serve as the first step in developing a roadmap to a sustainable market for biomanufacturing in space.

The symposium identified and prioritized multiple key R\&D opportunities to advance spacebased biomanufacturing. These opportunities fall in the areas of disease modeling, stem cells and stem-cell-derived products, and biofabrication. Additionally, symposium participants highlighted the critical need for additional data to help validate and de-risk these opportunities and concluded that approaches such as automation, artificial intelligence (Al), and machine learning will be needed to produce and capture the required data. Symposium participants also came to a consensus that public-private partnerships and funding will be needed to advance the opportunities toward a biomanufacturing marketplace in LEO.

This paper will summarize the current state of the science and technology on the ISS and in the fields of tissue engineering and regenerative medicine; provide an overview of biomanufacturing R\&D in space to date; review the goals of the Biomanufacturing in Space Symposium; highlight the key commercial opportunities and gaps identified during the symposium; provide information on potential market sizes; and briefly discuss the next steps in developing a roadmap to biomanufacturing in space.

\section{Introduction}

The use of LEO by governments and commercial enterprises is a complex ecosystem for providing opportunities and financing. In the last two decades, governments around the world, led by the U.S. and China, have heavily supported private space companies (2019 Report). These investments have focused on launch technologies, as high launch costs are perceived to be the greatest limiting factor to expanded space exploration and utilization (Werzt et al., 1996) and have led to recent reductions in the cost of transporting cargo to LEO by a factor of more than 20. Between 1970 and 2020, the average cost to launch a kilogram of payload into LEO on the space shuttle remained constant at about $\$ 54,500$. Now, the cost per kilogram is $\$ 2,720$ on a SpaceX Falcon 9 rocket (Figure 1) (Jones, H. W. et al., 2020). 


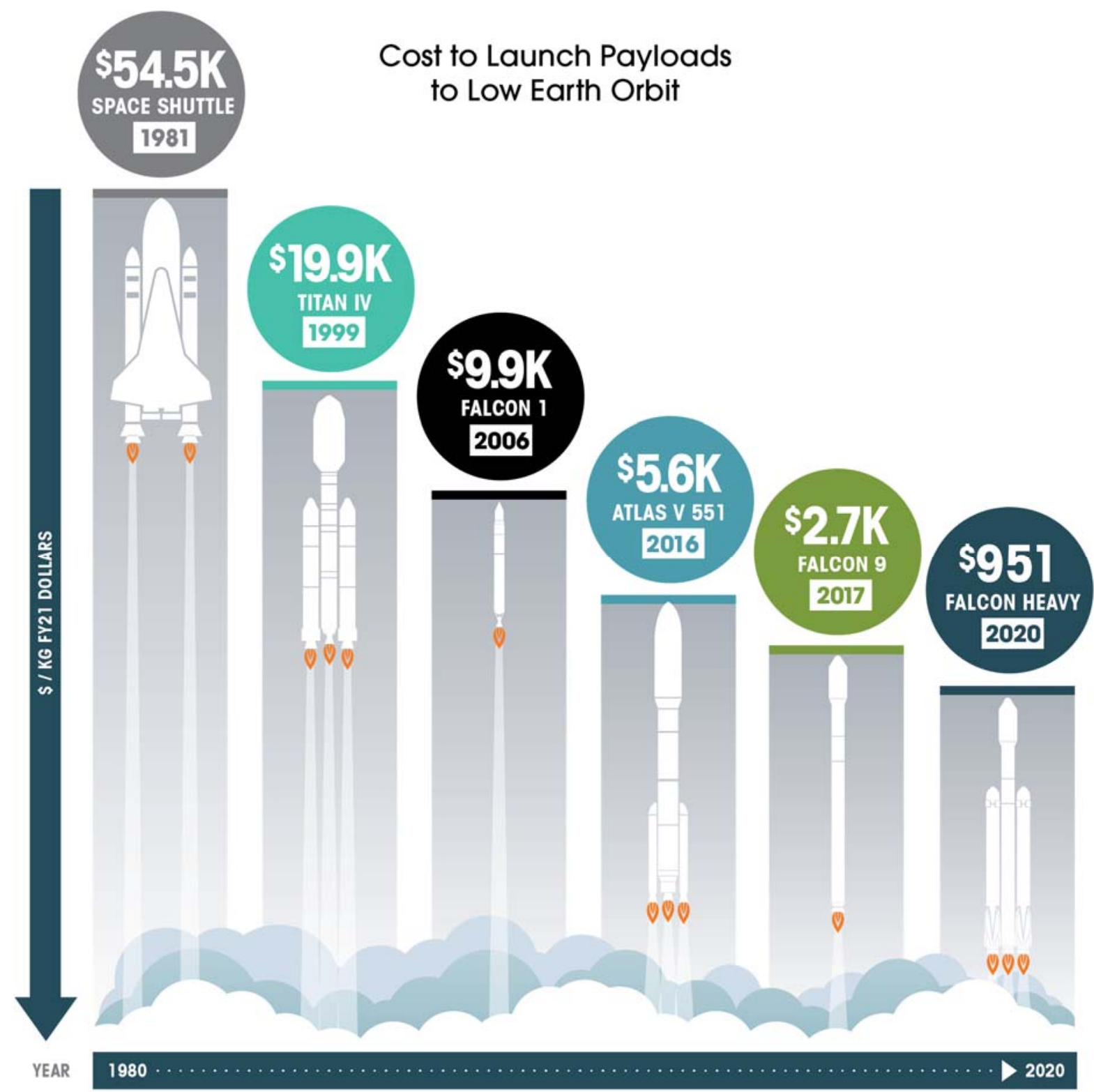

Figure 1: The cost of launching payloads to LEO has dropped considerably over the last 50 years. Note: Data is not to scale.

Additionally, several private companies are now pursuing commercial space stations. Axiom Space, headquartered in Houston, is currently developing what promises to be the first-ever privately operated space station, with the initial module scheduled to launch to the ISS in 2024. Axiom plans to dock multiple modules to the ISS that will eventually detach to become a standalone station. As the cost of transport to LEO has decreased-and is expected to decrease further-and plans for new platforms in LEO continue to advance (Dinkin S., 2019), opportunities in areas such as satellite deployment, biomedical research, in-space manufacturing, and space tourism increase. 
As the past half century has witnessed the opening of space for exploration and commercial opportunities, in this same period, we have experienced exponential growth in our understanding of biology and physiology. This knowledge has been translated and commercialized for the benefit of human health and continues to accelerate as new technologies create additional tools to explore and cure. One aspect of this biomedical revolution is in the field of regenerative medicine, built upon advances in stem cell biology, biomaterials, and bioengineering.

Remarkable advancements have been made in the design of MPS, also called tissue chips or organs-on-chips, and organoids that can mimic complex organ systems outside of the body for drug development or potential implantation to restore function. Stem cell isolation, characterization, and manipulation is advancing, with target applications broadly spread across tissues impacted by disease, trauma, and congenital conditions. Biomaterials and bioengineering advances have created new medical devices, targeted drug delivery platforms, biosensors and new imaging modalities, and the bioprinting of tissue constructs.

To take advantage of these significant advances-more frequent and more affordable access to LEO and exponential progress in biomedical technology - the question is: How do these intersect, and what new opportunities arise as both advance? How can the unique LEO environment be leveraged to further advance biomanufacturing? Compelling answers to these questions will introduce economic drivers for investment in space-based R\&D that extend beyond the initial focus on pure discovery and into the expansion of commercial development in LEO.

Over the past decade, the ISS National Lab has supported important space-based research in the areas of tissue engineering and regenerative medicine that lays the groundwork for more complex studies and future investment. This critical research addressed fundamental questions such as: How does the LEO environment affect the organ function mimicked by tissue chips, and how do these changes relate to human disease? How does microgravity affect stem cell proliferation and differentiation? And how might 3D bioprinting benefit from the absence of gravity?

Continued access to LEO through the ISS National Lab provides a unique opportunity for R\&D that enables the jump from this initial work to the development of a sustainable market for biomanufacturing in space. The ISS is a powerful platform with a limited lifetime and thus limited time left for utilization; therefore, now is the time to leverage this invaluable orbiting laboratory to conduct R\&D that demonstrates the value of biomanufacturing in space. This work will set the stage for increased private investment and the transition to larger and more numerous platforms in LEO that can support further discovery and development in the coming decades. 


\section{State of the Science and Technology}

\section{The International Space Station}

The ISS is arguably one of humankind's greatest engineering achievements (Figure 2). Assembly began in 1998 and has involved the U.S., Russia, Canada, Japan, and the participating countries of the European Space Agency in one of the most ambitious international collaborations ever attempted. Aside from the tremendous engineering accomplishment of constructing a football field-sized spacecraft in LEO, the ISS brings together the world's scientific research community to leverage the unique spaceflight environment for the benefit of humanity.

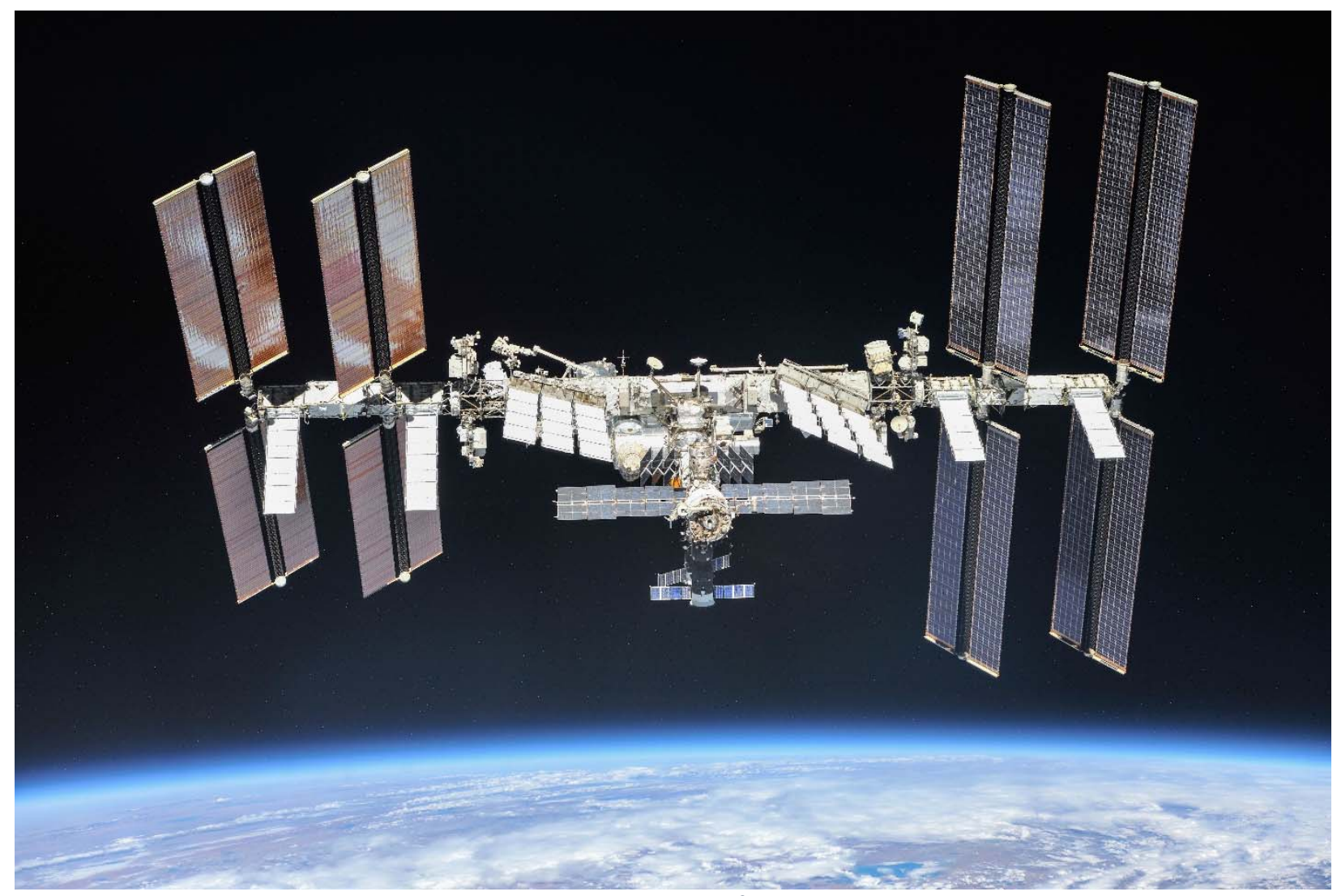

Figure 2: The International Space Station is a permanently crewed platform orbiting 250 miles above Earth. Credit: NASA

The ISS has already proven itself as a powerful platform for research and technology development. Over the more than 20 years of the station's life, more than 240 astronauts have conducted thousands of scientific experiments on topics ranging from water purification and cultivating plants to growing protein crystals and 3D printing human tissue. To date, the astronaut crews have invested nearly 50,000 hours in fundamental and applied research.

The R\&D activities on the ISS are generally categorized as benefiting space exploration or benefiting life on Earth, though there is overlap between these two application areas. NASA 
and the international partners leverage the ISS to validate technologies required for space exploration, making the ISS a critical test bed for life support systems, spacecraft systems, and the human body. The ISS National Lab user community leverages various aspects of the ISS environment to further R\&D to benefit life on Earth and advance commercial goals.

In the coming years, ISS experiments and facilities will increasingly be operated by the private sector. Dozens of commercial companies are already conducting R\&D on the ISS through the ISS National Lab. Additionally, a growing number of ISS National Lab Implementation Partners provide commercial services related to payload development in support of science investigations, and a subset of these partners (Commercial Service Providers) own and operate commercial facilities on the ISS.

Via NASA's Commercial Crew and Commercial Resupply Services contracts, commercial launch providers SpaceX and Northrup Grumman send crews and payloads to the ISS. Furthermore, NASA has selected and granted exclusive access to Axiom Space to attach modules to the ISS for a commercial space station that will ultimately become a privately operated standalone station (Figure 3). All this commercial activity serves to

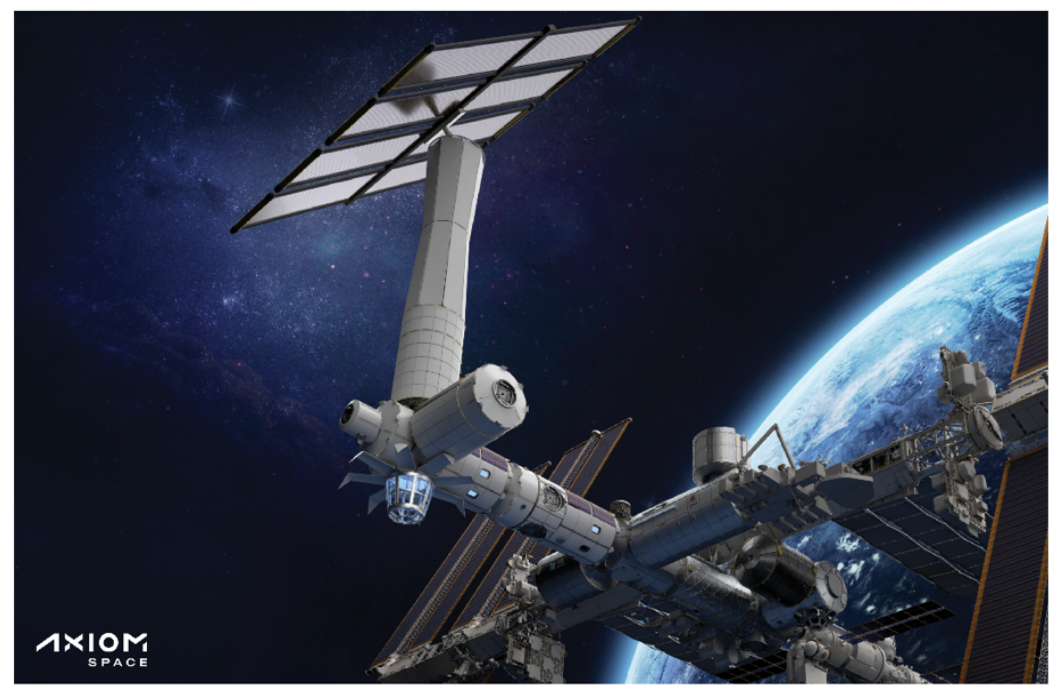

Figure 3: Artist's rendition of Axiom Station module 1 attached to the ISS (planned). Credit: Axiom Space pave the way for the development of a robust and sustainable economy in LEO.

\section{Tissue Engineering and Regenerative Medicine}

The fields of tissue engineering and regenerative medicine are poised to make a substantial impact on human health (Hunsberger, J. et al. 2000). While exciting, there are still substantial obstacles to overcome and knowledge gaps to fill in order to fully realize this potential (Chiao, M. (2017), Hunsberger, J., et al. 2015). The use of sustained microgravity has shown to provide potential benefits to overcoming some of these hurdles, from basic stem cell behavior to improvements in manufacturing processes.

\section{Stem Cells}

Stem cells hold great promise for use in therapeutic applications (Stern, J. H., et al. 2018, Rodríguez-Fuentes, D. E., et al. 2021, Ntege, E. H., et al. 2020, Sayed, N., et al. 2016) and as research tools (Sharma, A., et al. 2020). The ability to reprogram, differentiate, and gene edit stem cells, coupled with other advances in regenerative medicine tools, has led to a dramatic 
increase in uses for stem cells and stem-cell-derived products. Therapeutically, stem cells have been utilized to treat patients with cancers that affect the blood or immune system, such as leukemia and lymphoma (Master S., et al. 2019). Additionally, numerous studies are currently underway to test the efficacy and safety of stem cells for treating neurological disorders, autoimmune diseases, and injury to bone and skin (Chivu-Economescu, M., et al. 2017, Chlan L.L., et al. 2019, Phua, Q. H., et al. 2021).

The Alliance for Regenerative Medicine reports that in 2019 the global financing for cell and gene therapies reached nearly $\$ 10$ billion that year, and as of 2019 1,066 clinical trials were underway (Alliance for Regenerative Medicine, 2019). Stem cells have also experienced exponential growth as research tools, as evidenced by the 20 -fold increase in PubMed references cited from a key word search for "stem cell" from 1992 to 2020 (1,068 vs. 24,125, respectively) (Pubmed stem cell, 2021). Stem cells are being used for research that spans the spectrum from fundamental science to preclinical applications-including studies on human organ development (Yu, Q., et al. 2021), the underpinnings of aging (Kimmel, J. C., et al. 2021), and SARS-CoV-2-induced cardiac damage (Sharma, A., et al., 2000, Perez-Bermejo, J. A., 2021) as well as patient stratification for clinical trials (Wazir, U., 2020).

While there is tremendous potential in the use of stem cells and their derivatives for regenerative medicine applications, there are still several challenges confronting the field. $A$ LEO-based platform may deliver novel solutions such as improving expansion of somatic adult cells in vitro, allowing for improvements in genomic stability of induced pluripotent stem cells (iPSCs) during manufacturing, and improving self-assembly of organoids to promote more mature stem cell populations.

\section{Organoids and Microphysiological Systems}

Three-dimensional multicellular organoids and MPS that combine advances in microfabricated materials, tissue engineering, and cellular biology recapitulate the function of tissues and organs more accurately than standard two-dimensional cell culture tools (Low, L. A., et al. 2021). These relatively new technologies have seen a tremendous adoption in the research community over the past decade, with applications in disease modeling (Chan, A., et al. 2021, Donoghue, L., 2021), cancer diagnosis (Guo, Q. R., et al. 2021), drug safety and toxicity studies (Ma, L., et al. 2020, Fowler, S., 2020, Peterson, N. C., et al. 2020), and recently SARS-CoV-2 studies (Si, L., et al. 2021, Hysenaj, L., et al. 2021).

Commercial entities such as pharmaceutical, biotechnology, and cosmetic companies are turning to organoids and MPS as they look to develop tools that could help de-risk the development and validate the effectiveness of new products (e.g., therapeutics or cosmetics) (Low, L. A., et al. 2021). Pharmaceutical and biotechnology companies have begun implementing these technologies, demonstrating their usefulness in safety and toxicity profiling (Fowler, S., et al. 2020, Peterson, N. C., et al. 2020) as well as a number of other applications such a modeling the kidney (Phillips, J. A., et al. 2020), liver (Baudy, A. R., et al. 2020) and lungs (Ainslie, G. R., et al. 2019). The global MPS market size has been predicted to have a compound annual growth rate of more than $36 \%$ over the next five to six years (Valuates Reports 2021), 
driven by the adoption of the technology at commercial companies as well as the creation of specialized companies supporting the field through product development and contract services.

As the field grows, there are opportunities for which the use of a LEO-based platform could provide significant advantages; for example, the ability to model diseases using the accelerated aging effect experienced by humans in space. This unique feature could provide novel data and tools for the development of therapeutics to treat patients on Earth (Low, L. A., et al. 2019).

\section{Biofabrication}

Shortly after 3D printing technology was first introduced in the 1980s, medical professionals began exploring how such technology could be applied to fabricate tissue constructs with previously unachievable complexity, with the ultimate goal of developing functionality that would meet the standards for clinical application (Gu, Z., et al. 2020, Heinrich, M. A., 2019). Biofabricated tissue constructs could potentially utilize multiple types of the patient's own cells, reducing immunogenicity concerns and potentially allowing the construction of complex tissues comprised of architectures with distinct cellular organization. Biofabrication implementation models have envisioned the use of tissue assembly in situ (Lee, H., et al. 2021) as well as mass production approaches.

Over the past decade, since the first commercially available 3D bioprinter was developed, exploration into possible applications for this technology has grown exponentially. Nearer-term target products include biofabricated organoids or miniature tissue analogs for disease modeling and therapeutics testing, biofabricated patches for accelerated wound healing, devices made with conductive bioinks, and food production (Yi, H. G., et al. 2021, Varkey, M., et al. 2019, Mandrycky, C., et al. 2016, Djisalov, M., et al. 2021). Longer-horizon ambitions that have captured the public's imagination include complete organ printing, with on-demand creation of personalized organs. Such advances would revolutionize the treatment of end-stage organ failure and eliminate the reliance on limited donor organs and the chronic immunosuppression associated with transplantation.

In addition to the 3D bioprinting hardware and control software, critical components in the biofabrication process include the selection of cell type(s), carrier fluids, and scaffold components. Intrinsic to each printing strategy is the development of mechanical stability from what is usually a fluidic "ink" to a self-supporting construct. This can occur using chemical reaction or phase change early after the print placement process. Bioinks commonly integrate cells with carrier fluids that include proteins and other biocompatible materials. A printed tissue construct may be designed for immediate use or implantation or provided the necessary time and conditions for maturation before use.

By utilizing the microgravity environment, biofabrication on a LEO-based platform could offer several unique advantages. For example, biofabrication in microgravity has the potential to eliminate the need for rapid structural stabilization after printing. This could allow the use of bioinks with lower viscosity and a reduced or eliminated requirement for crosslinking or other scaffolding (Moldovan N. I., 2018). These unique features of microgravity could enable novel 
biofabrication designs and improved methods of maturation (Gungor-Ozkerim, P. S., et al. 2018, Zhang, S., 2018).

Additionally, microgravity conditions could enable the development of new types of biofabrication technologies not possible on the ground, such as devices for freeform bioprinting. Furthermore, biofabrication in LEO could provide organoids and tissues for disease modeling and therapeutics testing in microgravity. Thus, space-based biofabrication has the potential to be useful not only for manufacturing new, high-quality products but also as a tool that could complement opportunities identified in the stem cell and organoid/MPS research areas.

\section{Overview of Biomanufacturing in Space}

\section{Benefits of Space-Based Biomanufacturing}

Over the past decade, the ISS National Lab has supported numerous space-based studies in the areas of tissue engineering and regenerative medicine (Figure 4). This initial R\&D has provided important

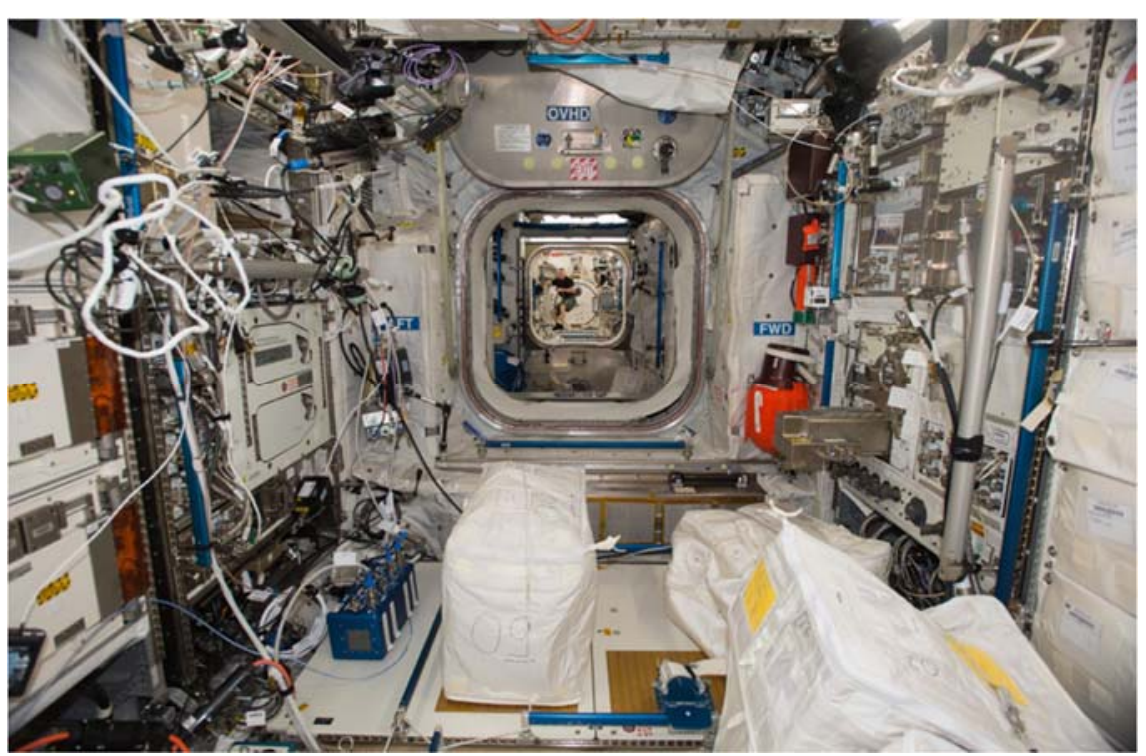

Figure 4: The ISS provides a unique platform in sustained microgravity to conduct R\&D in ways not possible on Earth. Credit: NASA insights into how microgravity can be leveraged to advance biomanufacturing in space to benefit human life and commercial enterprise on Earth. Microgravity induces changes in body systems that result in effects that include cardiovascular deconditioning, skeletal muscle atrophy, bone loss, and immune dysfunction, among others. These effects mimic the onset of health-related outcomes associated with aging and chronic human disease but at an accelerated rate-effects that could take years to manifest on Earth may develop in weeks in microgravity (Vernikos, J., et al., 2010, Patel S., 2020, Smith J. K., 2020, Shelhamer, M., et al. 2020). While these changes are a concern for keeping astronauts safe on long-duration spaceflight, they also present an opportunity to study aging and disease progression and test therapeutics on an accelerated timescale. For this reason, researchers have begun utilizing MPS on the ISS to create accelerated models of aging and disease for the development of therapeutics for Earth-based patients (Low, L. A., et al. 2019).

Microgravity also alters gene expression and DNA regulation and causes changes in cellular function and physiology. Removing the effects of gravity has contributed significantly to the 
collective fundamental knowledge of cellular behavior, cell-cell interactions, tissue development and regeneration, and aggregate interactions in the context of a whole organism (Grimm, D., et al. 2014, Garrett-Bakelman, F. E., et al. 2019, Giulianotti, M. A., et al. 2019, Bradbury, P., et al. 2020, Herrmann, M., et al. 2020, da Silveira, W. A., et al. 2020). Pioneering bioengineering experiments on the ISS coupled with ground-based studies have demonstrated that microgravity enables the study of novel features not attainable under normal gravity conditions, including changes to stem cell proliferation rates (Baio, J., et al. 2018, Yuge, L., et al 2006) and differentiation (Blaber, E. A., et al. 2015, Jha, R., et al. 2016, Imura, T., et al. 2019).

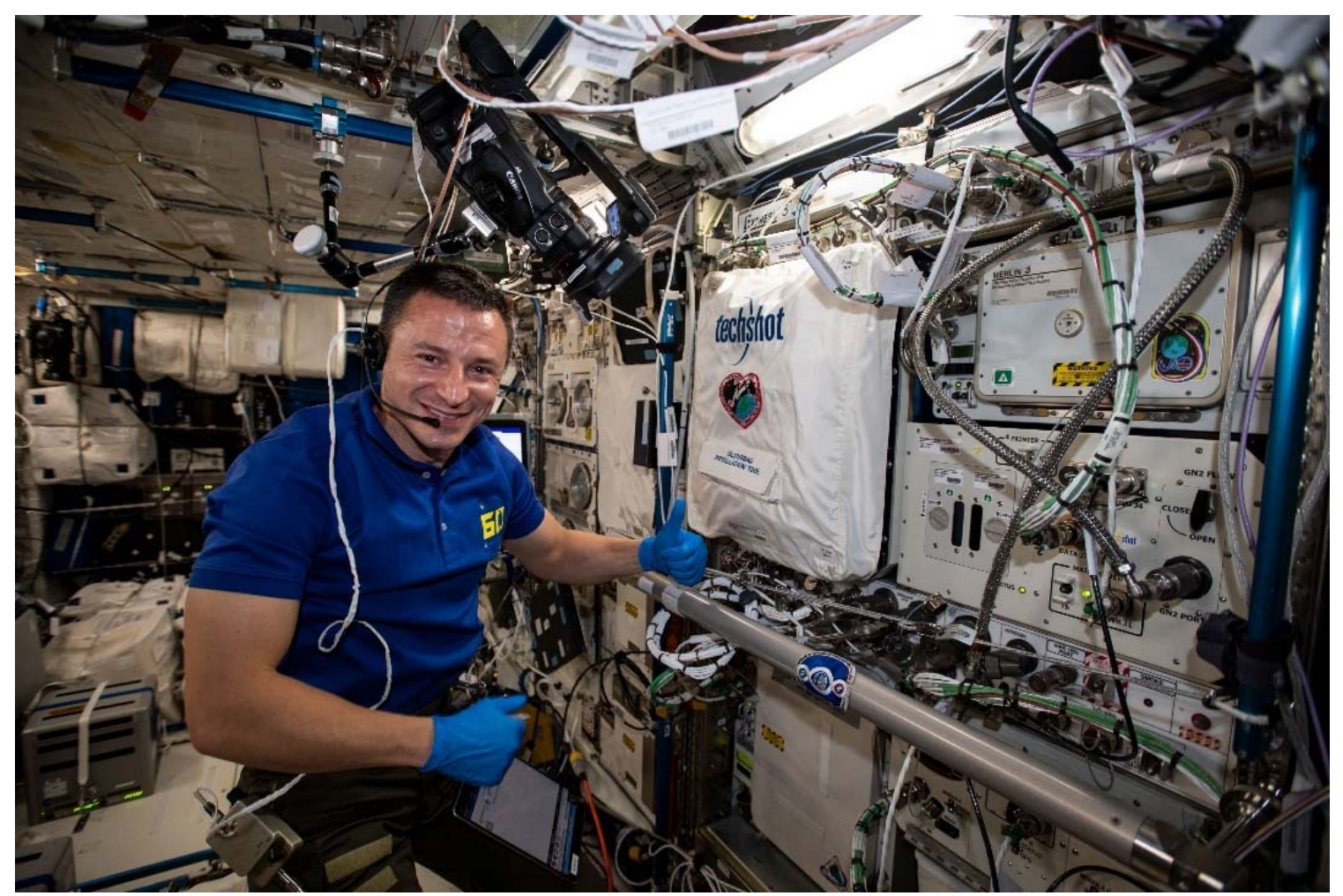

Figure 5: NASA astronaut Andrew Morgan sets up Techshot's BioFabrication Facility to test-print tissues onboard the ISS. Credit: NASA

Additionally, bioprinting tissues in microgravity provides potential advantages in the use of lower viscosity biomaterials or bioinks and the ability to fabricate diaphanous biological structures. The processes involved in biofabrication are heavily reliant on biomechanical and mechanobiological cues that are affected by gravity, and microgravity conditions should enable full control over these cues in ways not possible on Earth. A sustained microgravity environment also allows for scaffold-free tissue engineering, enabling researchers to overcome what has been a significant hurdle in ground-based biofabrication of soft human tissue (Prasad, B., et al. 2020). 
There are currently two commercial bioprinters on the ISS: the Russian 3D Bioprinting Solutions Organaut, which utilizes magnetic particles to create clusters of cells, and the American BioFabrication Facility (Figures 5 and 6), which directly dispenses bioink through four different print heads. The BioFabrication Facility, from Techshot, Inc., has successfully completed test prints of a partial human meniscus and is now being used to create test prints of cardiac-like tissue of increasing thickness.

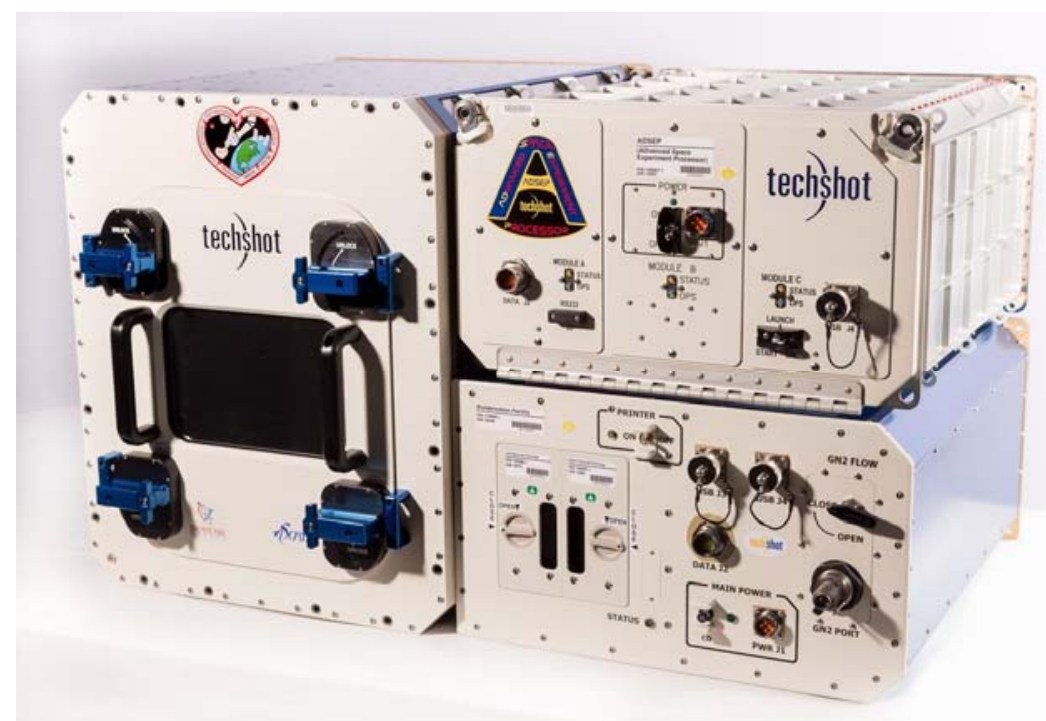

Figure 6: Techshot's BioFabrication Facility and accompanying Advanced Space Experiment Processor unit. Credit: Techshot

Microgravity can also improve biofabrication processes that involve thin-layer deposition, through which thin film layers of biomaterial are deposited onto a substrate material with atomic-level precision. The reduction in gravity-driven forces such as sedimentation and buoyancy enable dominant surface tension effects to produce more uniform and precise layering. High-precision thin-layer deposition enabled by microgravity could have significant value in the production of medical devices.

\section{Current Biomanufacturing R\&D on the ISS}

In recent years, the ISS has seen increased utilization by commercial, academic, and government users focused on leveraging microgravity for research and product development with Earth-based benefits (Giulianotti, M. A., et al. 2019). A number of government agencies other than the National Aeronautics and Space Administration (NASA), such as the National Institutes of Health $(\mathrm{NIH})$, the National Science Foundation (NSF), and the Department of Defense (DoD), have engaged in multiyear funding initiatives that utilize the ISS National Lab (Giulianotti, M. A., et al. 2019, Low, L. A., et al. 2019).

NIH's National Center for Advancing Translational Sciences (NCATS) and National Institute of Biomedical Imaging and Bioengineering (NIBIB) have collaborated with the ISS National Lab on the Tissue Chips in Space initiative, a multiyear, multiflight initiative to leverage MPS platforms in microgravity to advance understanding of human disease, with the goal of translating 
findings into potential new therapeutics for patients on Earth (Figures 7-9). NSF has also partnered with the ISS National Lab on a multiyear initiative to fund studies that utilize the ISS for advancements in the field of transformative tissue engineering within the scope of the NSF Engineering of Biomedical Systems Program, including research in the areas of cellular engineering, tissue engineering, and modeling of physiological systems.

Studies funded by NIH and NSF through these initiatives have included space-based MPS

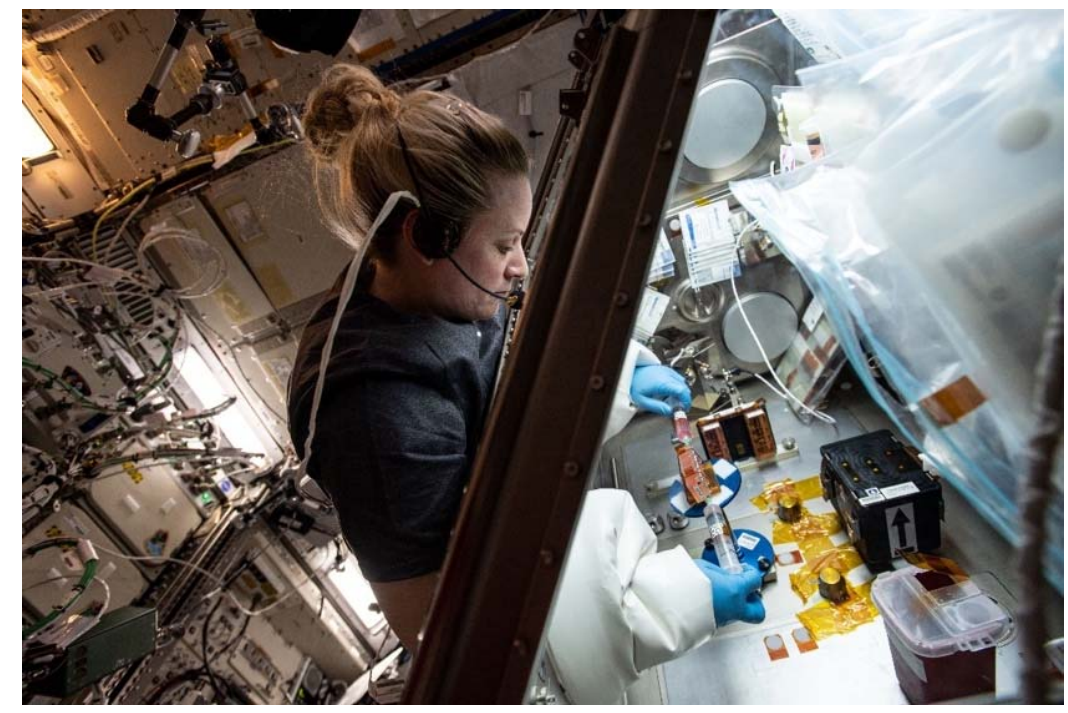

Figure 7: NASA astronaut Kate Rubins works on the Cardinal Heart investigation, funded by NIH through the Tissue Chips in Space initiative, that evaluated whether three-dimensional engineered heart tissue exposed to sustained microgravity conditions displays characteristics similar to ischemic cardiomyopathy. Credit: NASA research to model kidney proximal and distal tubule physiology, cartilage-bone-synovium joint interactions, blood-brain barrier physiology, liver aging and immune response, and cardiac muscle tissue, among others. Representatives from both NIH and NSF participated in the Biomanufacturing in Space Symposium.

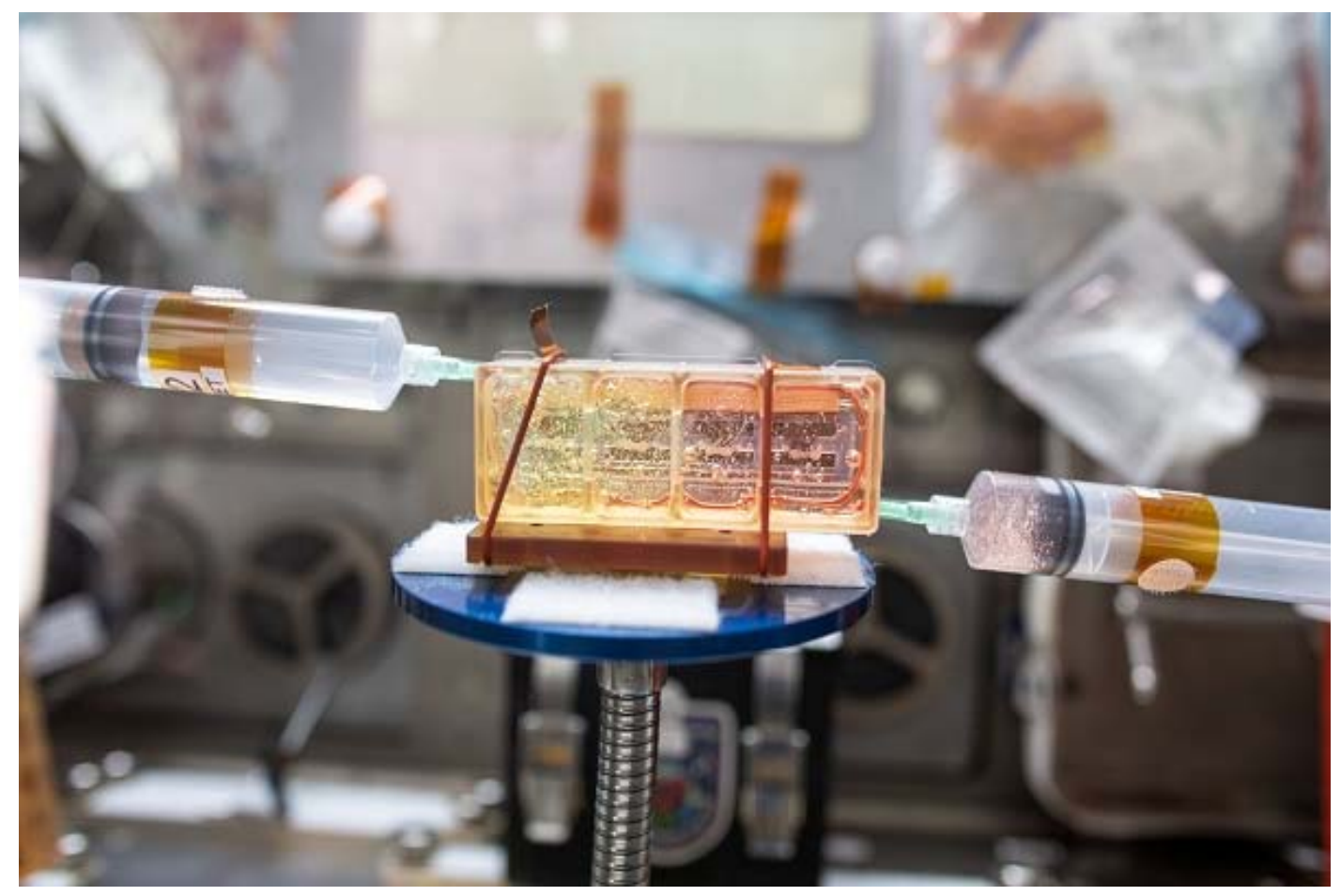

Figure 8: An astronaut (out of frame) adds RNAlater to a gas permeable tissue chamber to preserve engineered heart tissue constructs for the Cardinal Heart investigation. Credit: NASA 
In addition, an increasing number of pharmaceutical and biotechnology companies are using the LEO environment to validate drug targets and test the efficacy of potential therapeutics on accelerated models of disease (Cadena, S. M., et al. 2019, Giulianotti, M. A. et al. 2019). For example, Novartis used a rodent model to explore aging and muscle atrophy, and Emulate, Inc. used a proprietary innervated Intestine-Chip system to examine immune response to diseasecausing bacteria.

Additionally, Merck \& Co. has utilized the ISS to examine new crystallization methods to improve the manufacturing, storage, and delivery of pembrolizumab, the active agent in the immuno-oncology drug Keytruda ${ }^{\circledR}$ (Reichert, P., et al. 2019). In microgravity, where sedimentation and convection currents are minimized, Merck was able to produce highly uniform, stable concentrated crystalline suspensions of pembrolizumab and translate findings to improve ground-based drug formulations.

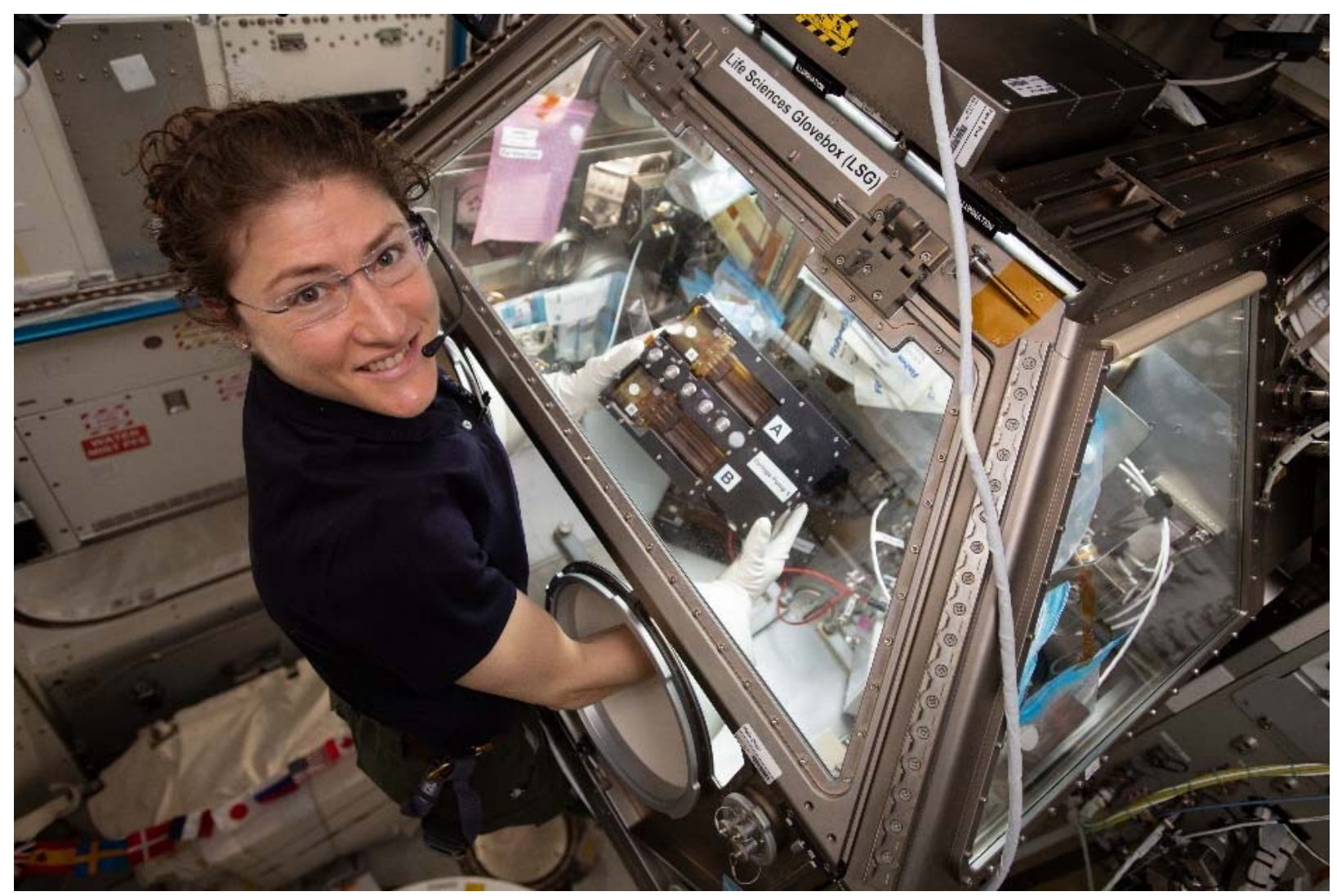

Figure 9: NASA astronaut Christina Koch works on a Tissue Chips in Space investigation that used a proximal and distal tube tissue chip system to study kidney function. This investigation utilized hardware from BioServe Space Technologies. Credit: NASA

Smaller startup companies are also finding value in space-based R\&D. Biotechnology startup LambdaVision is using the ISS to test in-space biomanufacturing processes to advance development of an artificial retina that restores vision in patients with retinal degeneration. The artificial retina is produced through layering of a light-activated protein, and microgravity improves the uniformity of the layers. 


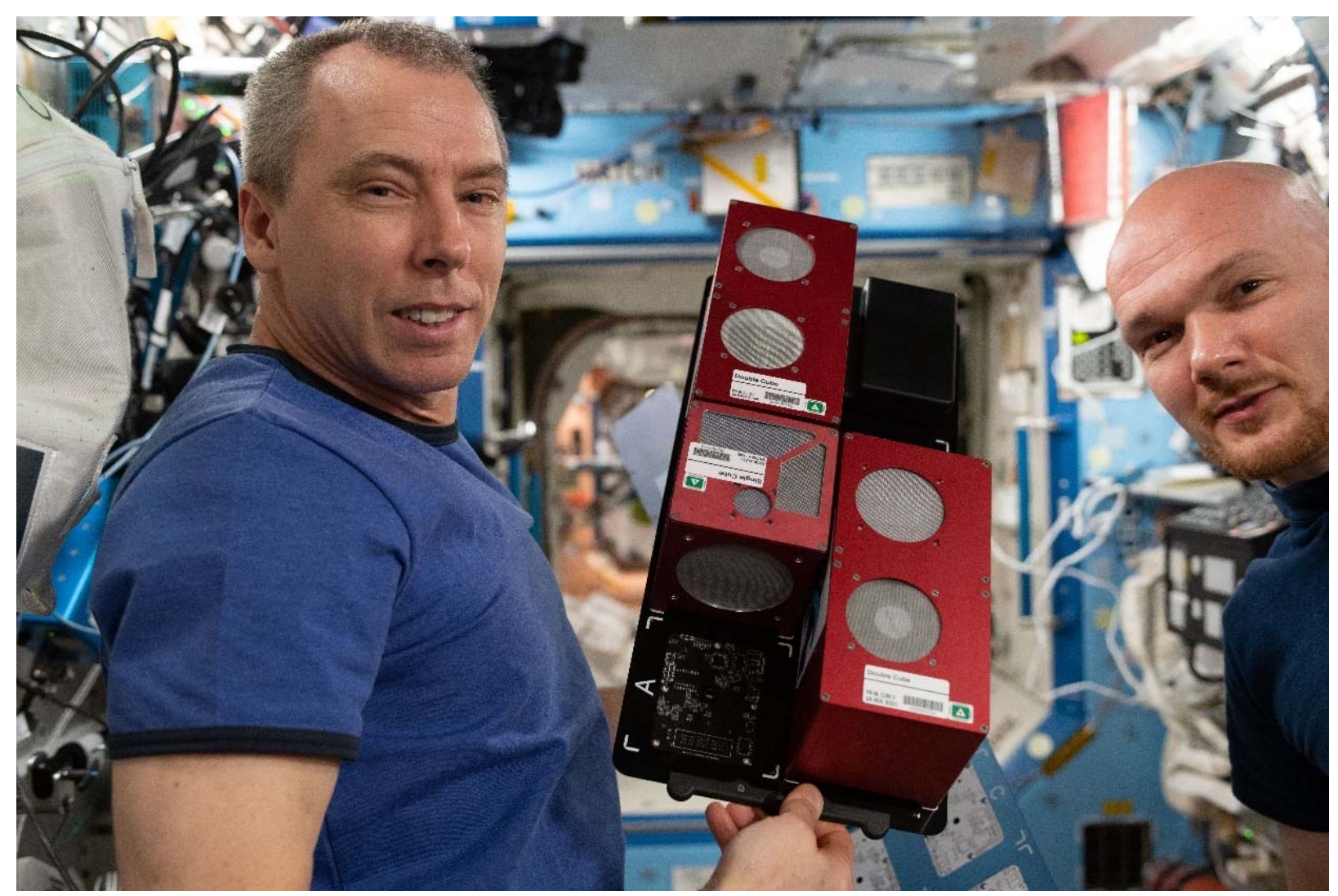

Figure 10: NASA astronauts Drew Feustel and Alexander Gerst with Space Tango's TangoLab hardware onboard the ISS. Credit: NASA

Meanwhile, NASA and the ISS National Lab are working with a growing number of Commercial Service Providers, organizations that actively develop and operate ISS facilities and equipment that enables space-based biomedical R\&D (Figure 10) (Implementation Partners 2021). Several of these Commercial Service Providers-BioServe Space Technologies, Techshot Inc., and Space Tango-participated in the symposium and shared valuable perspectives and knowledge of ISS research, including areas of terrestrial research and manufacturing that could benefit from access to space.

In parallel, commercial companies such as Axiom Space, Blue Origin, Sierra Space, and Space Tango, among others, are designing vehicles, satellites, and other platforms that will enable future opportunities in space-based biomanufacturing R\&D. This ongoing work will ensure a smooth transition from ISS-based pilot studies to full-scale biomanufacturing in LEO.

It is critical for current and future stakeholders to work collaboratively to identify the best opportunities to utilize the ISS now for R\&D that will enable a robust in-space biomanufacturing market in the future. The ISS has a limited lifetime, and the amount of research that can be sent to the ISS and conducted through the use of ISS crew time is also limited. Thus, the opportunities must be prioritized such that the economic value of LEO-based research can be clearly demonstrated, allowing for further investment and growth based on this success. 


\section{Biomanufacturing in Space Symposium}

CASIS and the McGowan Institute for Regenerative Medicine at the University of Pittsburgh hosted a Biomanufacturing in Space Symposium to serve as the first step in developing a roadmap to a sustainable market for biomanufacturing in space. The symposium included a series of virtual workshops, presentations, and interactive discussions with internationally recognized subject matter experts in the areas of tissue engineering and regenerative medicine as well as space-based R\&D. The goal was to identify the most promising opportunities to leverage the ISS to advance space-based biomanufacturing. Specifically, opportunities identified needed to focus on the development and translation of commercially relevant biomolecules and biomaterials for use in preclinical, clinical, and therapeutic applications.
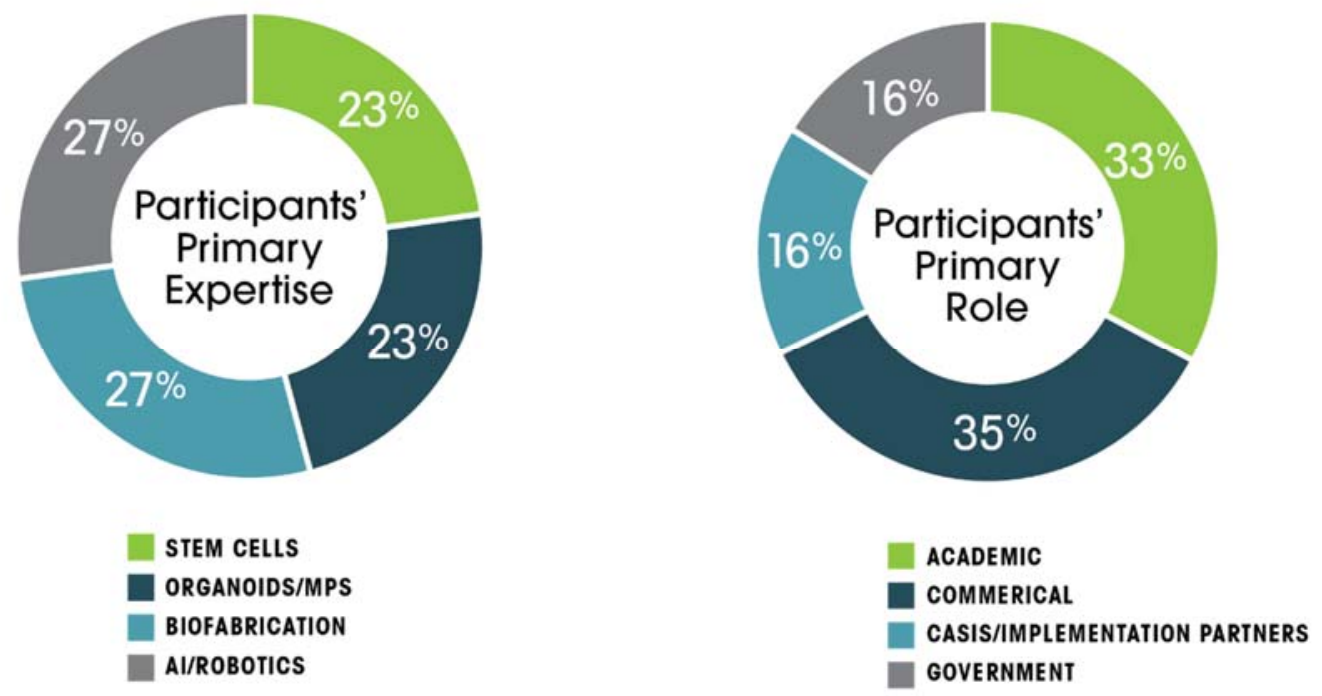

Figure 11: Breakdown of symposium participants' expertise and primary role.

Originally planned as a two-day in-person event, the Biomanufacturing in Space Symposium was moved to a virtual format that took place over several months, primarily during the last quarter of 2020. The symposium's 138 participants represented a diverse background of expertise, which significantly increased cross-education and learning (Figure 11). The symposium had three topic areas: stem cells, organoids and MPS, and biofabrication, and working sessions were divided into three phases: educate, generate, and prioritize.

During the educate phase, subject matter experts gave presentations on the topic areas and the stages to commercialization, to prepare symposium participants for cross-domain discussions. The generate phase consisted of a series of sessions in each topic area that sought to identify the most promising opportunities to leverage the ISS to advance space-based biomanufacturing and highlight current gaps and commercial opportunities. The prioritize phase was aimed at converging the key opportunities identified in each of the topic areas and outlining the next steps in developing a roadmap for biomanufacturing in space. 
A separate session during the symposium explored how automation, $\mathrm{Al}$, and machine learning could be integrated to enable iteration of science and enhance the R\&D process in LEO.

In the following section, we present the key R\&D opportunities identified and prioritized as well as the gaps and commercial opportunities.

\section{Key Opportunities Identified}

During the generate phase, more than 50 potential commercial opportunities across the three tracks were discussed in detail. Convergence of the most promising opportunities across the tracks was critical, and during the prioritize phase, the opportunities were prioritized using direct feedback from symposium participants based on the following three factors:

- Role of microgravity: The importance of microgravity in enabling the opportunity.

- Impact: The attractiveness of the opportunity for investment, including the magnitude of impact and concentration of investors.

- Risk: The risk associated with the opportunity, measured by risk of failure, time to market, and risk-benefit tradeoff.

The most promising opportunities identified through the symposium fell into three areas: (1) disease modeling, (2) stem cells and stem-cell-derived products, and (3) biofabrication. Below highlights the key opportunities in each of these areas, including the scientific rationale, current gaps, and commercial opportunities.

\section{Disease Modeling}

Across all three tracks, symposium participants extensively discussed the opportunity to utilize a sustained microgravity environment for disease modeling-whether through the use of stem cells, organoids, MPS platforms, or tissues either biofabricated in orbit or assembled terrestrially and brought to space. Participants highlighted the unmet need for novel approaches to model disease and aging (Low, L. A., et al. 2021), and several researchers have utilized the ISS as a platform to develop disease models based on the physiological changes associated with spaceflight (Low, L. A., et al. 2019).

Participants agreed that the opportunity to uniquely isolate the stresses induced by sustained microgravity could provide significant insights into the aging process and disease progression. This opportunity area is supported by decades of extensive research into the effects of spaceflight on human physiology, model organisms, and cell biology. Data from these spacebased studies indicate that humans experience significant physiological changes during adaptation to spaceflight and during readaptation upon return to Earth (Luxton, J. J., et al. 2021, Garrett-Bakelman, F. E., et al. 2019, Meck, J. V., et al. 2001, Akima, H., et al. 2000). Below, we highlight some of the promising disease modeling opportunities identified through the symposium. 


\section{A Note on Food and Drug Administration (FDA) Considerations}

During the symposium, participants discussed how regulatory considerations might influence biomanufacturing in space. Participants generally agreed that, ultimately, early conversations with the FDA are critical. Many of the future space-based biomanufacturing opportunities discussed involve the production of materials for human therapeutic use, and the use of LEO may introduce several potential areas of risk that would need to be carefully considered.

However, the primary goal of the symposium was to identify potential commercial opportunities and begin to develop a roadmap for realizing them. At this early stage, the need was to broadly consider potential applications rather than attempting to detail specific regulatory risks. The symposium included participants with experience either working for the FDA or working directly with the FDA, and some of the unique risks were informally noted (e.g., radiation exposure, high g-force exposure, etc.).

Symposium participants also noted that FDA concerns surrounding trackability, quality assurance, and quality control would apply to both space-based and terrestrial-based manufacturing facilities. Participants also noted that the aerospace industry has substantial expertise in the areas of trackability, quality assurance, and quality control. Finally, participants also agreed that interactions with the FDA should be part of refining a roadmap for biomanufacturing in space.

\section{Specific Examples}

\section{Muscle Wasting}

On Earth, muscle wasting occurs due to several root causes, including disuse, age, and underlying disease. Impaired muscle mass and function not only significantly affect quality of life but also represent a substantial economic burden, with direct healthcare costs in the U.S. estimated to be more than $\$ 18.5$ billion in 2000 (Janssen, I., et al. 2004). Since then, these costs have continued to increase considerably (Beaudart, C., et al. 2014). As the aging population increases, muscle wasting is becoming an even greater public health concern. The U.S. Census Bureau has projected that the population aged 65 and older will reach more than 83 million people by 2050, almost doubling the estimated population of 43 million in 2012 (Ortman, J., et al. 2014).

Research has shown that during spaceflight, humans lose skeletal muscle at an accelerated rate, and countermeasures are required to dampen the accelerated loss (Vernikos, J., et al. 2010). Thus, microgravity-induced muscle loss provides an opportunity to study muscle wasting progression on a faster time scale than is possible on Earth. Multiple studies have been conducted using rodents in microgravity as an accelerated disease model to elucidate the 
mechanisms underlying muscle atrophy and to test new potential therapeutics (Lawler, J. M., et al. 2021, da Silveira, W. A., et al. 2020, Semple, C., et al. 2020, Chakraborty, N., et al. 2020, Smith, R. C., et al. 2020).

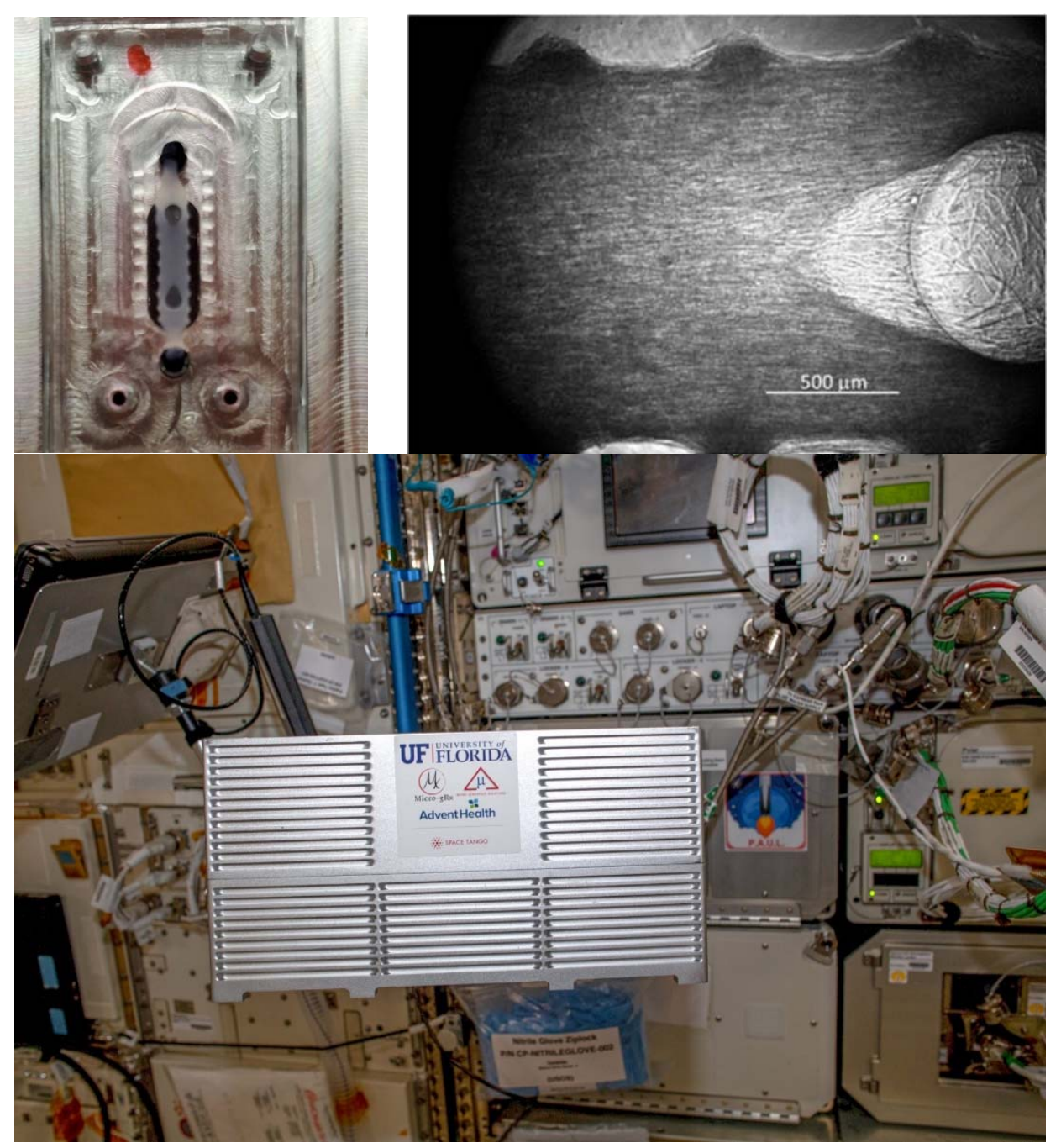

Figure 12: Engineered skeletal muscle tissue in a microfluidic chip pressed onto a fluid manifold prior to spaceflight (top left) and a 10x image of the same tissue in orbit captured by a microscope built into the autonomous CubeLab (top right). Credit: Generated by the Siobhan Malany Laboratory at the University of Florida in collaboration with Space Tango. Photo of the CubeLab onboard the ISS (bottom). Credit: NASA

Moving beyond rodent models, microgravity provides a unique opportunity to study sarcopenia and disuse atrophy in a human cellular model. Earth-based rodent studies can be done using hind-limb suspension to model disuse; however, the constant force of gravity makes it impossible to mimic disuse in human cellular models on Earth. Thus, utilizing a LEO-based platform to study myocytes from different patient populations could uniquely enable the development of models for drug target identification and therapeutic evaluation (Figure 12). Such research could reveal druggable pathways that may not otherwise have been uncovered in terrestrial studies. 


\section{Changes in Cardiac Physiology}

Cardiovascular disease, the leading cause of death in the U.S., is responsible for one in four deaths (Virani, S. S., et al. 2020). It is also a major economic burden, with the U.S. cost of cardiovascular disease-related health care services, medication, and lost productivity totaling around \$219 billion per year from 2014 to 2015 (Fryar, C. D., et al. 2012).

In microgravity conditions, humans experience acute changes in cardiac physiology, structure, and function (for example, the heart remodels to assume a more spherical shape due to altered cardiac loading from reduced gravitational forces), and long-term microgravity exposure leads to cardiac deconditioning. Due to this cardiac deconditioning, orthostatic intolerance is evident in astronauts upon returning to normal gravity (Lee, S., et al. 2015). Arrythmias have also occurred in astronauts during spaceflight, even in those with no prior history of arrythmias (Delp, M. D., et al. 2016). However, the acute cardiac changes associated with spaceflight (Smith J. K. 2020) largely return to baseline after return to normal gravity. Furthermore, studies indicate that astronauts on missions lasting six months to one year do not have an increased rate of developing cardiovascular disease after return to Earth (Ade, C. J., et al. 2017).

Thus, a LEO-based platform could enable the modeling of an acute, microgravity-induced cardiovascular phenotype and the reversal of this phenotype through the use of twodimensional stem-cell-based models, three-dimensional tissue-engineered constructs, organoids, or MPS models. Such research could aid in elucidating the mechanisms of cardiovascular function and enable improved disease modeling and therapeutic screening.

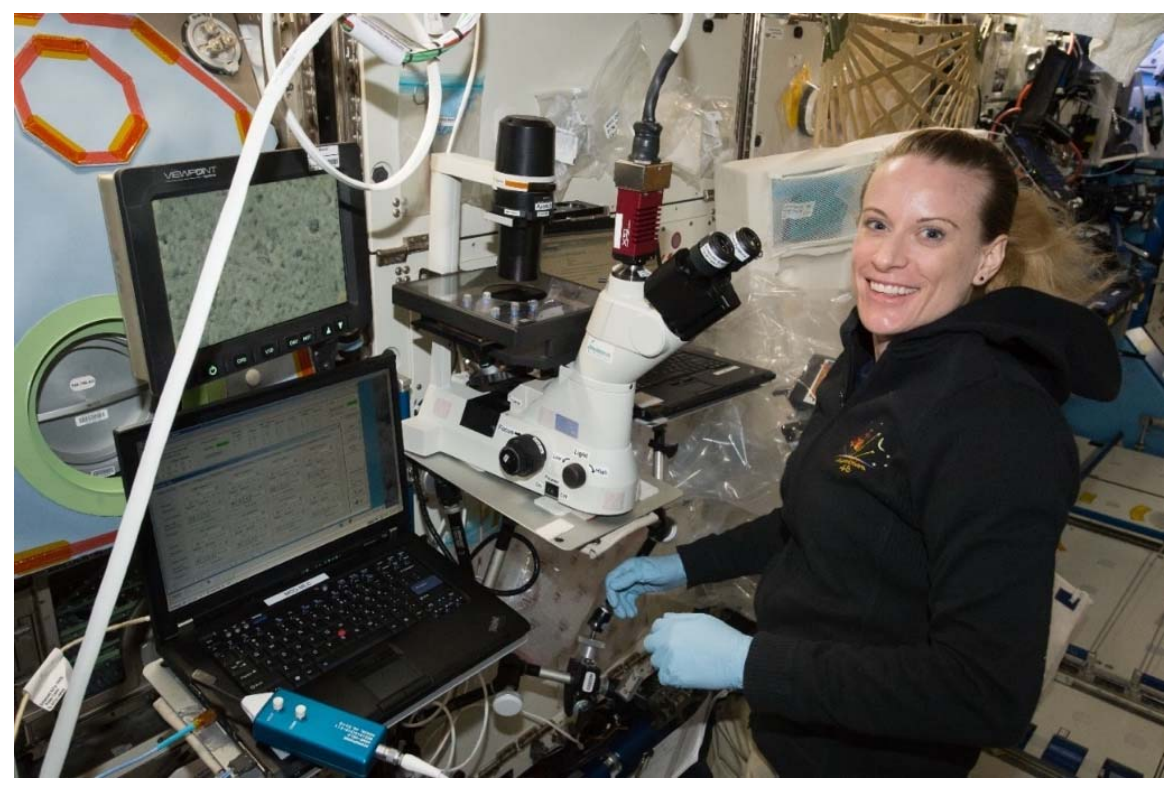

Figure 13: NASA astronaut Kate Rubins examines cardiac cells onboard the ISS as part of an investigation studying the effects of microgravity on stem-cell-derived cardiac cells. Credit: NASA

Preliminary work on the ISS (Figure 13) has demonstrated the successful culture and return of viable two-dimensional cardiomyocytes derived from iPSCs and found that microgravity alters cardiac function at the cellular level, with changes in cell contraction and gene expression 
mirroring those observed in astronauts during spaceflight (Wnorowski, A., et al. 2019). Additional studies are currently in progress utilizing multilineage, tissue-engineered cardiac constructs to study the impact of microgravity on cardiac physiology and function (Low, L. A., et al. 2019). Two different studies are examining engineered heart tissues formed from human cardiomyocytes differentiated from iPSCs (Figures 7, 8, and 14).

Figure 14: NASA astronaut Jessica Meir onboard the ISS working with engineered heart tissues in the final spaceflight hardware. This investigation is examining the effects of microgravity on human cardiac tissue structure and physiological function. Credit: NASA

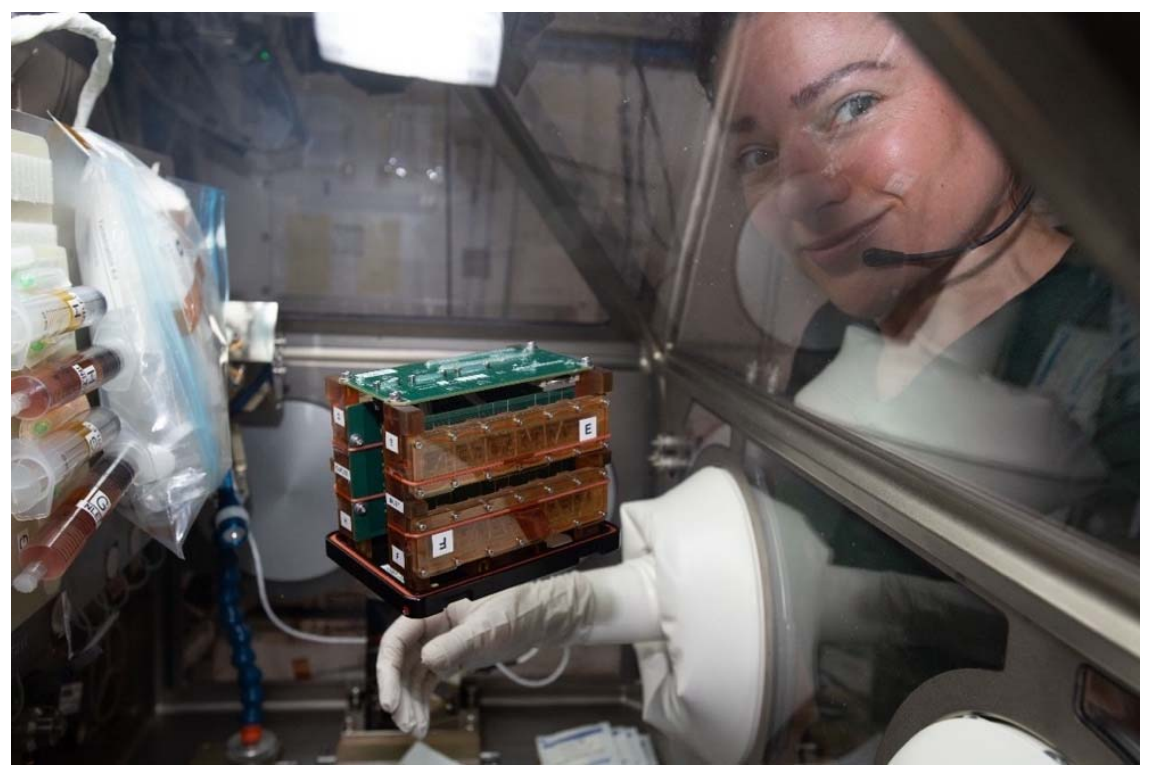

\section{Osteoarthritis}

Osteoarthritis - a painful condition in which the cushioning and lubricious cartilage at the ends of bones breaks down-affects hundreds of millions of people worldwide and is one of the most widespread diseases that lacks successful drug treatments. While there are effective drugs for rheumatoid arthritis, the much more prevalent osteoarthritis has no approved disease-modifying therapeutics, only short-acting pain relievers that do not halt or even slow the progression of disease (Yusuf, E. 2016) In older patients, osteoarthritis accounts for a large proportion of the estimated 600,000 knee replacement surgeries done in the U.S. each year. Post-traumatic osteoarthritis, a chronic condition that can develop after a traumatic joint injury (such as an ACL or meniscus rupture), represents approximately $12 \%$ of the osteoarthritis population and similarly has no approved disease-modifying therapeutics for treatment (Thomas, A. C., et al. 2017, Bajpayee, A. G., 2017).

In microgravity conditions, humans experience accelerated bone loss, and extended spaceflight can alter bone integrity in a fashion roughly analogous to osteoarthritis. In addition, the effects of altered loading of joint cartilage in space may affect the extent and rate of cartilage breakdown leading to osteoarthritis. The use of MPS or other tissue systems on a LEO-based platform could enable accelerated disease modeling, and such studies may provide unique insights into disease progression and uncover novel targets for therapeutic interventions to treat osteoarthritis. 
Furthermore, microgravity uniquely enables the study of disuse versus exercise in the management of osteoarthritis and post-traumatic osteoarthritis. Modeling disuse in human cellular models is not possible on Earth; however, microgravity can enable studies using human cells and tissues to examine the effects of not only disuse but also applied mechanical force. Carefully controlled mechanical forces could be applied to tissues to mimic different types of exercise in conjunction with therapeutics. Studies are currently underway on the ISS utilizing a post-traumatic osteoarthritis MPS model to identify novel pathways to treat the condition and test therapeutic interventions (Low, L. A., et al. 2019).

\section{Aging}

The medical community has an intense interest in aging research that aims to prolong healthy and productive years. Novel approaches to enhance quality of life include targeting cellular senescence (Tchkonia, T., et al. 2021), genomic instability (Li, Z., et al. 2021), and telomere shortening (Li, Z., et al. 2021). Studies have shown that spaceflight induces several physiological changes in both astronauts and rodent models, including skeletal muscle atrophy, bone density loss, immune dysfunction, cardiovascular deconditioning, and arterial stiffening, among others.

These changes, which resemble aging-related maladies on Earth, occur rapidly during spaceflight and are mostly reversible upon return to Earth. This provides a compelling case for leveraging microgravity conditions to improve understanding of aging and related disease processes. MPS models, organoids, or biofabricated tissues exposed to microgravity and then returned to Earth could provide significant insight into novel biological targets associated with disease progression and regression as well as anti-aging.

Several space-based studies have been done on aging-for example, one of the projects supported through the NCATS Tissue Chips in Space initiative (Tissue Chips in Space 2021) is leveraging an MPS model for immunological senescence in microgravity to investigate the relationship between immune aging and tissue healing as well as regenerative capacity (Low, L. A., et al. 2019). Additionally, NASA's One-Year Mission on the ISS with NASA astronaut Scott Kelly found that the average telomere length in Kelly's white blood cells increased during the mission and then returned to preflight levels after his return to Earth. However, in the months following his spaceflight mission, a greater number of his telomeres were lost or critically shortened (Garrett-Bakelman, F. E., et al. 2019). These findings could present a therapeutic target that could be studied in the context of a LEO-based model, as telomere shortening and loss are associated with aging and susceptibility to age-related diseases, including cardiovascular problems and cancer.

\section{Biofouling}

Medical devices are prone to surface biofouling, which results from nonspecific adhesion of proteins, cells, and microorganisms. Complications associated with such fouling include blood clot formation and embolization as well as device-centered infections. These phenomena are associated with a substantial degree of morbidity and mortality across several types of 
implantable medical devices. Biofilms form on wetted surfaces during spaceflight (Zea, L., et al. 2020), and on the ISS, the formation of microbial communities in the form of biofilms is often accelerated. Thus, while not specifically disease modeling, a LEO-based platform could be used to accelerate the timeline for biofouling characterization of biomaterials and elucidate mechanisms that may be altered in microgravity in ways that increase or decrease virulence. In fact, studies have shown that during spaceflight, some bacterial strains appear to exhibit increased virulence (Simões, M. F., et al. 2021).

\section{Gaps}

One of the primary current gaps noted during the symposium is the existing need for validation of space-based disease models for terrestrial applications. This is not a unique concern, as validation is always a primary issue when developing new models of disease. The question is always how clinically relevant the models are and how information obtained from the models may be utilized in therapeutic development (Low, L. A., et al. 2021). These answers could be addressed in part by increased data and throughput, which was also noted as a current gap.

The use of LEO for modeling terrestrial diseases is at a relatively nascent stage, and the ability to do large-scale experiments in the LEO environment is limited by the challenges of launching and conducting experiments in space. Symposium participants agreed that continued advancements in miniaturization, automation, the implementation of Al and machine learning, and the standardization of equipment (hardware), biological materials, and protocols would increase the opportunity to generate meaningful data. Of note, these are all key areas where improvements in technologies for use in space will readily translate to and benefit terrestrialbased applications.

Another key area for improvement that participants highlighted is the ability to rapidly iterate on experimental results, which is currently limited by the inherent logistical challenges of performing experiments in space. This gap could be addressed by having an inventory of inorbit supplies such as banked cells and pre-seeded devices or organoids to provide the ability to biomanufacture models in space as needed. Additionally, increased throughput and data acquisition could be accelerated further as launch frequency continues to increase.

\section{Commercial Opportunities}

One commercial opportunity identified during the symposium is in the data surrounding novel targets for therapeutic development. Participants discussed several ways in which this opportunity could be realized. The formation of a syndicate of pharmaceutical and biotechnology companies could serve to de-risk early-stage common opportunities. This would lower the investment risk for any individual stakeholder and create the infrastructure for future single investments, with the tradeoff being distributed control of any intellectual property developed through the syndicate. There are established precedents for these types of partnerships, such as the IQ Consortium-a syndicate of pharmaceutical and biotechnology companies that collaborate to make meaningful advancements in the biopharmaceutical industry. 
Another opportunity lies in collaborations with other government agencies and foundations that have interests in specific diseases that could be modeled in LEO. This would build on the work pioneered by NCATS through the Tissue Chips in Space Initiative but with a focus on specific diseases or goals. Participants also expressed enthusiasm about working with space agencies (NASA as well as international agencies such as Japan Aerospace Exploration Agency and the European Space Agency), commercial space companies, and other government agencies to find common areas that could inform both risk reduction in space exploration and advances in human health on Earth.

\section{More Effective Stem Cells and Stem-Cell-Derived Products}

Stem cells and stem-cell-derived products are promising as both research tools (Sharma, A., et al. 2020) and therapeutic products (Stern, J. H., et al. 2018, Rodríguez-Fuentes, D. E., et al. 2021, Ntege, E. H., et al. 2020, Sayed, N., et al. 2016). Symposium participants discussed the potential to leverage a LEO-based platform to gain insights into how to control and optimize stem cell pluripotency and multipotency, proliferation and expansion, genomic and epigenomic integrity, differentiation, and maturation. This opportunity area is supported by published work demonstrating that sustained microgravity influences the behaviors of stem cells and stem cell derivatives. Below, we highlight some of the promising opportunities related to stem cells and stem-cell-derived products identified through the symposium.

\section{Specific Examples}

\section{Cells With Increased Potency and Expansion Capabilities}

Stem cells are defined by their potency, or their ability to give rise to multiple derivative cell lineages. Pluripotent stem cells are able to transform into all cells of the body except for placental tissues. Multipotent stem cells can differentiate into cells of a specific lineage; for example, hematopoietic stem cells give rise to various cell types of the blood and immune system.

However, a major challenge in the field is variability in stem cell potency from cell line to cell line, accompanied by an inability to maintain potency and genetic integrity as cells proliferate. Thus, it is critical to identify novel methodologies that will either maintain or enhance the potency, quality, and differentiation capacities of stem cell lines. Such improvements in cell characteristics would have an impact on the tissue engineering and regenerative medicine industries in both R\&D and therapeutic applications. The symposium highlighted potential therapeutic applications that already have preliminary spaceflight data, including the following.

- Creating cells and tissues for cardiac repair: Following cardiac injury in the human heart, regeneration is limited. Human cardiac cells are largely non-proliferative, with less than $1 \%$ of cardiomyocytes replaced yearly (Bergmann, O., et al. 2015). With insufficient ability to replace damaged or apoptotic cardiomyocytes, myocardial injury 
results in replacement of the damaged tissue with fibrotic scar tissue (Travers, J. G., et al. 2016). Methods to enable proliferation of cardiomyocytes and subsequent cardiac regeneration are being actively investigated, along with the implications of microgravity for cardiac regeneration. Studies have found that cardiac progenitor cells cultured on the ISS exhibited increased proliferative and migratory potential due to changes in mechanotransduction pathways and, subsequently, cytoskeletal organization (Baio, J., et al. 2018, Camberos, V., et al. 2019). This is supported by works using simulated microgravity on Earth (Jha, R., et al. 2016). Determining the molecular foundations of these genotypic and phenotypic responses could allow for proliferation and migration of healthy cardiomyocytes into areas of cardiac injury, resulting in regeneration rather than maladaptive remodeling. This initial work in sustained microgravity conditions indicates that such an environment can lead to the identification of novel targets for enhancing the therapeutic benefit of cardiovascular stem cells or progenitor cells (Camberos, V., et al. 2021, Camberos, V., et al. 2019, Baio, J., et al. 2018).

- Expansion of mesenchymal stem cells (MSCs) with improved clinical properties: While stem cells hold great potential for use as therapeutic agents, safe and efficient expansion of stem cells is still a major challenge in the field (Zhang, Y., et al. 2021). MSCs are emerging as the most common stem cell type utilized within the field of regenerative medicine (Research and Markets, 2021). Recent spaceflight studies indicated that human MSCs can be grown safely on the ISS and that they have improved immunosuppressive capabilities compared with MSCs cultured on Earth (Huang, P., et al. 2020). Additionally, MSCs cultured under simulated microgravity conditions showed increased therapeutic potential in a traumatic brain injury model (Otsuka, T., et al. 2018).

\section{Stem Cell Differentiation}

Symposium participants also discussed the unique stimulus that a sustained microgravity environment can confer on stem cell differentiation, including differentiation of iPSCs. The discussion ultimately centered on two primary themes: (1) what we could learn from an indepth characterization of stem cell differentiation conducted in space and (2) whether microgravity could allow for the generation of cell types not currently possible from terrestrially based ex vivo differentiation of stem cells, including differentiation of iPSCs.

- Characterizing stem cell differentiation in microgravity: Terrestrial-based studies have demonstrated that differences in culturing conditions and cell source can have significant impacts on stem cell differentiation (Yim, E. K., et al. 2012). While several studies have shown different effects to stem cells cultured in sustained microgravity (Imura, T., et al. 2019), it is difficult to extrapolate results from these studies due to the incongruent nature of the conditions, equipment, and cell sources utilized. There is a need to fully characterize how microgravity as a variable influences stem cell differentiation into the three primary germ layers (mesoderm, ectoderm, and 
endoderm). A comprehensive side-by-side analysis of stem cell differentiation in LEO compared with ground conditions utilizing the current state-of-the-art conditions and analysis techniques should be performed.

- Generation and expansion of hematopoetic stem cells (HSCs) from iPSCs: HSCs have been used clinically as stem cell therapies for decades. However, clinical utility is limited by the reality that the number of in-need patients exceeds the population of matching donors, coupled with limited success in optimizing ex vivo expansion (Carreras, P., et al. 2021, Walasek, M. A., et al. 2012). One potential solution for these issues could be the ability to generate HSCs from iPSCs (Wahlster, L., et al. 2016). Experiments in sustained microgravity could uncover pathways to enable this process and thus allow for the production of HSCs in a manner that overcomes the supply limitations. Spaceflight studies also have the potential to identify culture conditions for expanding and maintaining the potency of HSCs from cord blood or other nonembryonic tissues, which could provide another means to address the shortage of donor HSCs for bone marrow transplantation.

\section{Gaps}

During the symposium, the primary gaps identified for these opportunities centered around the current lack of data and standards. These are points in which much can be learned from terrestrial-based research efforts, where a lack of data and agreed upon standards. To address these issues, the International Stem Cell Initiative (ISCI) has established standards for assessing pluripotency of iPSCs (International Stem Cell Initiative 2018), and the International Society for Stem Cell Research (ISSCR) publishes guidelines to promote best practices (Daley, G. Q., et al. 2016). The community engaging in LEO-based stem cell R\&D should align with current best practices in order to ensure space-based results are translatable across both spaceflight and terrestrial studies.

Similarly, symposium participants discussed the importance of the origin and source of cells and the subsequent effects on results. Participants agreed that, in general, more than one donor source-and, in fact, as many as possible or feasible-should be utilized, and the donor sources should be widely available. A village-in-a-dish approach could be applied (Mitchell, J. M., et al. 2020) to maximize the diversity of the populations studied.

Currently, a lack of data is an inherent issue to LEO-based research in this area due to the challenge of conducting R\&D in space; however, by adopting standards and applying highthroughput approaches, miniaturization, microfluidics, robotics, machine learning, and Al, the quality and quantity of data return could be dramatically amplified. Moreover, many of the technology advancements required to enable stem cell research on a LEO-based platform would readily translate to terrestrial applications, such as the development of compact, robust, high-throughput systems capable of working autonomously and remotely. 


\begin{abstract}
A Note on Simulated Microgravity
Due to the challenging and costly nature of conducting spaceflight experiments, several approaches have been utilized to simulate or model the microgravity environment on Earth. For the cell culture and bioengineering fields, these approaches include clinostats, rotating wall vessels, random positioning machines, and rotary cell culture systems (Benavides Damm, T., et al. 2014, Grimm, D., 2018, Herranz, R., et al. 2013)

While these systems allow one to manipulate the effects of Earth's gravity on a given sample, they do not fully recapitulate the sustained microgravity conditions on a LEO-based platform such as the ISS (Ferranti, F., er al. 2020, Wuest, S. L., et al. 2015). As with any model system, care must be taken not only to observe differences in results produced through the use of simulated microgravity devices but also to design the experiments so that differences among the various types of devices and between simulated and true microgravity conditions can be unambiguously demonstrated to be causal rather than coincidental (Wuest, S. L., et al. 2015, Acres, J.M., et a. 2021).

Additionally, depending on the design of the simulated microgravity device, the total specimen volume that experiences a microgravity effect is limited to $\mathrm{mm}$ - to $\mathrm{cm}$-sized volumes. However, on a LEO-based platform, specimen volume is theoretically only limited by the volume available to culture the given samples. Even with these limitations, it is important to consider the utility and cost efficiency of simulated microgravity devices as part of the spectrum of microgravity tools in order to provide additional insights, validate hypotheses, and augment data generation (Topal, U., et al 2021, Krüger, M., et al. 2019, Imura, T., et al. 2019, Grimm, D., et al. 2018).
\end{abstract}

\title{
Commercial Opportunities
}

Stem cells and stem-cell-derived products have two primary customer bases: (1) those who utilize stem cells as research tools and (2) those who utilize stem cells in therapeutic applications. Symposium participants generally agreed that in the near term, the largest value return on investment will be derived from the data that can be translated to improving terrestrial processes, products, and tools. However, the participants felt that, ultimately, largescale production of certain types of stem cells and stem-cell-derived products could benefit from manufacturing in a LEO-based facility and that the LEO environment could confer certain advantages that may not be replicated in a terrestrial setting.

Symposium participants agreed that in the near term, this area of research would benefit from deliberate interactions with government agencies. The use of the unique LEO environment could provide key insights that may have substantial impacts to the larger research community and thus would be of great public interest. Publicly funded R\&D is necessary to move beyond the current roadblocks and make commercial opportunities more attractive. As specific use cases emerge demonstrating that large-scale, in-space biomanufacturing provides the potential 
for return on investment, increased commercial engagement will follow. To foster and prepare for these potential opportunities, discussions with cell-based therapeutic manufacturers, commercial space station developers, the research community, and the FDA should begin now. These interactions should focus on the infrastructure needed to scale from LEO-based research facilities to LEO-based manufacturing facilities and on identifying the requirements needed to enable FDA approval. Finally, symposium participants highlighted that in our current economy, where data is currency, there is an opportunity for private funding aimed at securing the unique intellectual property and data generated from conducting stem cell research in space.

\section{Biofabrication}

During the symposium, biofabrication discussions covered a wide variety of opportunities, including fabricating tissues for disease modeling (discussed above), testing and maturation of biofabricated materials, and improved fabrication processes for biomaterials and biofabricated constructs. With commercial companies having recently invested in technology development for terrestrial and in-space biofabrication, discussions at the symposium provided an overview of current efforts and a glimpse of future opportunities. Below, we touch on some of the promising opportunities identified through the symposium.

\section{Specific Examples}

\section{Thin-Layer Deposition}

The process of assembling thin films through layer-by-layer deposition is of significant interest for applications such as optics, membranes, sensors, biomedicines, and several energy-related applications (Richardson, J. J., et al. 2015, Gentile, P., et al. 2015). In recent years, studies have focused on scaling up processes for thin-layer deposition to enable real-world application (Richardson, J. J., et al. 2015). The current terrestrial processes for layer-by-layer assembly are influenced by the materials being used and the number of layers being deposited, which can affect the overall integrity of the product being generated. Specifically, gravity-driven sedimentation of elements with differing densities can influence the speed at which new layers can be deposited as well as the ordering of molecular components into biologically functional assemblies. The influence of gravity on each layer is additive, so potential issues that may occur in the layering process are compounded as the number of layers in the construct increase.

Sustained microgravity could be beneficial by potentially enhancing the production quality of products manufactured through thin-layer deposition. Reducing the influence of gravity-driven forces such as buoyancy and sedimentation on the surface tension and homogeneity of the solutions or materials used for deposition could allow for more uniform layering and a higherquality multilayer (Dag, O., et al. 1997). For example, studies are underway to test the use of microgravity conditions on the ISS to improve the manufacturing process of artificial retinas produced via thin-layer deposition. 
In addition to deposition processes, microgravity may also improve self-assembly processes involving components with varying sedimentation characteristics. Furthermore, microgravity could provide benefits for solidification processes, such as with metallic materials, where the precipitation of undesirable phases or inclusions might be minimized. In medical applications, these benefits could provide improved ductility for alloys in devices such as stents.

\section{Tools for Biofabrication}

There is an urgent need for tissues, organs, and other biomaterials for use in transplantation and regenerative medicine applications (Hunsberger, J., et al. 2015, Ntege, E. H., 2020). In the past decades, several novel biofabrication tools have emerged to enable the assembly of complex structures (Pedde, R. D., et al. 2017). All of these tools either integrate or attempt to circumvent the effects of gravity in their biofabrication processes. Given the broad demand for additional biofabrication tools and techniques, discussions during the symposium centered on potential advantages that biofabrication in space might confer.

For example, sustained microgravity could enable the use of less viscous bioinks and reduce reliance on chemical and physical crosslinking strategies for rapid structural stabilization that must be applied in concert with printing. Microgravity could also provide the ability to enhance cell-cell interactions for organoid production, the ability to control mechanotransduction effects due to gravity during the maturation/curing process, and the ability to print simultaneously from any spatial orientation.

Two commercially operated biofabrication systems currently reside on the ISS: the Techshot BioFabrication Facility and the Russian 3D Bioprinting Solutions Organaut. The BioFabrication Facility (BioFabrication Facility 2021) has successfully produced test prints of a partial human meniscus and will next produce test prints of cardiac-like tissue of increasing thickness.

Symposium participants agreed that there are opportunities to utilize these current biofabrication systems on the ISS and to develop new systems that take advantage of sustained microgravity conditions, enabling unique approaches to biofabricate materials such as tissue constructs.

\section{Gaps}

Biofabrication is a relatively recent addition to the ISS research portfolio. Early identification of gaps will support effective guidance toward more efficient development of the field. However, the challenge is to do so in a way that also leaves room for innovation and discovery. Initial discussions regarding gaps centered on the need to gain additional insights on fundamental behaviors of materials (i.e., cells, liquids, and proteins) in a sustained microgravity environment and to determine how such information could influence the design and utilization of biofabrication approaches (Ahari, H., et al. 1997, Dag, O., et al. 1997, McPherson, A., et al. 2015). Additionally, given that biofabrication processes would occur remotely (on unmanned platforms in LEO) with communication delays of greater than seconds, the need for robust automation linked to machine learning was also a focal point of discussion. 
For biofabrication processes that show promise, and where commercial opportunities are identified (such as in the manufacturing of artificial retinas), participants raised questions concerning scalability, quality control, intellectual property, and regulatory approval. For all these points, participants agreed there are parallels to terrestrial applications, and lessons learned from both ground- and space-based R\&D will further the field collectively. For example, fundamental understanding of material behavior is central to the utility of those materials used in biofabrication processes regardless of location of fabrication. Furthermore, the ability to develop biofabrication systems that generate reproducible products in a scalable manner that would satisfy regulatory approval is critical for not only space-based processes but also terrestrial-based processes. To assuage some of these concerns, participants noted that Axiom Space is planning for modular facilities in its commercial space station, where compartments can be isolated from the rest of the station to enable quality-controlled manufacturing.

\section{Commercial Opportunities}

Symposium participants identified two primary opportunities to enable the further development of space-based biofabrication applications: public funding and private investment. The collective need for novel approaches to produce implants, tissues, and organs is a public concern, and there are opportunities for government agencies to put resources into utilizing a unique environment such as a LEO-based platform to advance the field. There are also opportunities to explore the fabrication of advanced biomaterials that may have applications outside of regenerative medicine that might be of interest to agencies such as the Department of Defense or others.

For applications where commercial opportunities are identified, symposium discussions centered on private investment and the challenges around investors' desire for short timelines and a multiplier for return on investment. Participants agreed that private investment in specific applications had potential if risk-versus-return valuations could satisfy investors. Currently, the LEO economy is dynamic and fluid. Private investment has begun in some areas such as spacecraft and in-orbit platforms. Investment in other areas, including space-based biomanufacturing, may follow. Furthermore, as more commercial companies become involved in developing spacecraft and in-orbit platforms, there will likely be a continued reduction in the costs and time associated with LEO-based manufacturing.

\section{Automation, Artificial Intelligence, and Machine Learning}

A common theme that emerged in the working groups throughout the symposium is that more data are needed to help validate and identify the most valuable scientific and commercial opportunities that the LEO environment confers. The current lack of data is due in no small part to the challenging nature of accessing LEO and conducting research in a space-based

environment. Symposium participants consistently noted that utilizing advances in automation, $\mathrm{Al}$, and machine learning could enable an exponential production of the data needed to make informed decisions. 
Currently, tools are being developed to automate terrestrial cell culture (Cohen-Karlik, E., et al. 2021) and biofabrication methods (De Pieri, A., et al. 2021). These automated technologies could be applied to research in the LEO environment, allowing experiments to run autonomously and potentially scale in the progression from research to clinical applications (Vieira, C. P., et al. 2021). This approach would enable researchers to continuously generate data and test conditions in a manner that reduces the need for astronaut intervention (a currently limited resource) and associated astronaut training. Automation would also facilitate intellectual property protection, as closed systems could be devised to protect confidential or proprietary work steps.

Additionally, research groups are applying machine learning and Al to improve cellular products (Mota, S. M., et al. 2021, Cohen-Karlik, E., et al. 2021), biomaterial manufacturing (An, J., et al. 2021, Lee, J., et al. 2020), and disease modeling (Gao, D., et al. 2021) (Figure 15). Utilizing existing data sets, both from terrestrial experiments as well as LEO-based experiments (da Silveira, W. A., et al. 2020), machine learning approaches could be built into the automated LEO platforms. As new data are generated, more advanced Al approaches could further be utilized to focus on the specific applications where LEO confers advantages over terrestrialbased facilities.

\section{Traditional iterative development pathway}

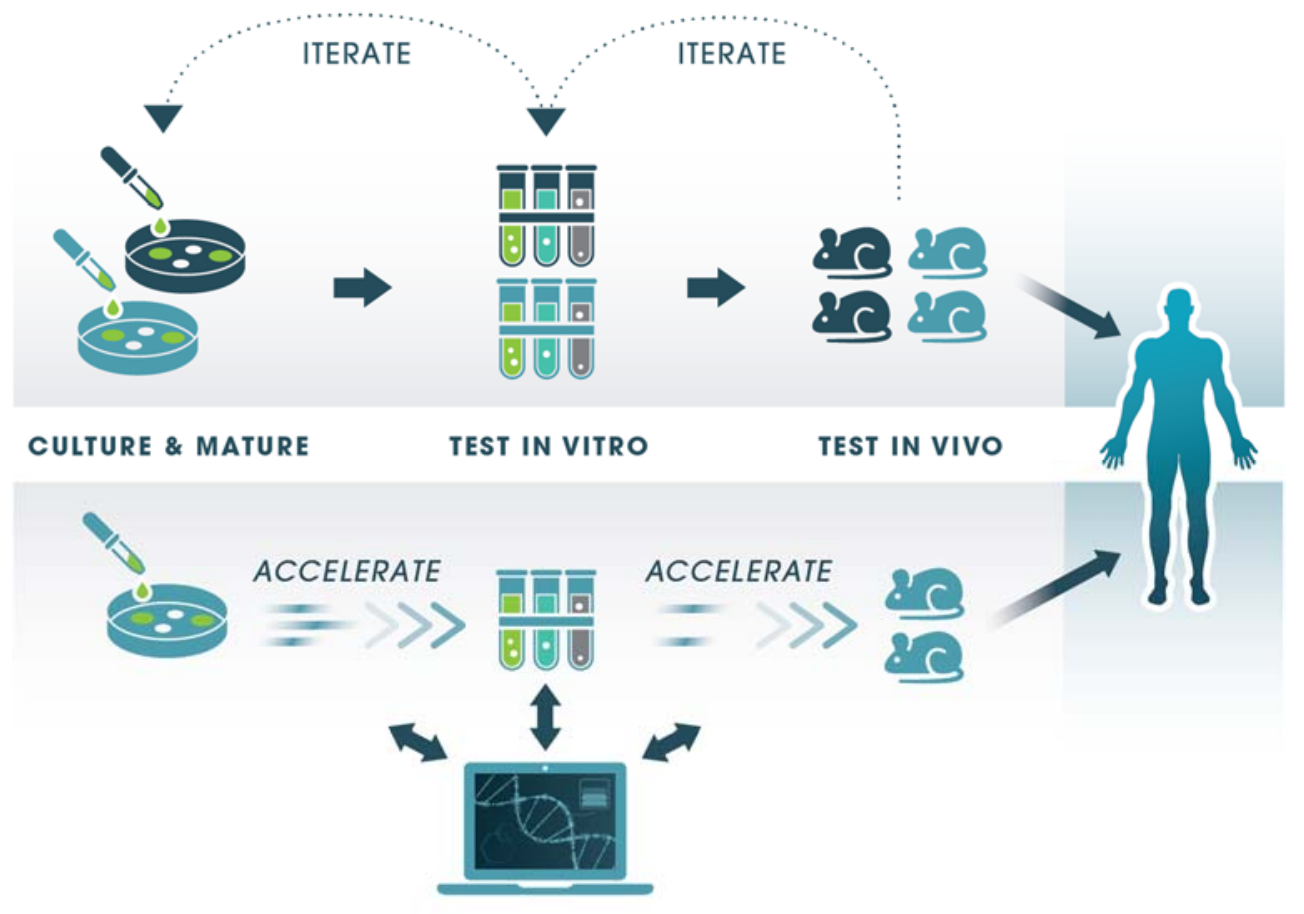

\section{Accelerated in silico development pathway}

Figure 15: Development pathways integrated with automation, $M L$, and Al can accelerate the process and utilize less resources. 
Additional data are needed to de-risk space-based biomanufacturing and make informed decisions about future directions of the field. Symposium participants agreed that automation, $\mathrm{Al}$, and machine learning will be necessary to produce these data. Use of these approaches would improve R\&D cycles to better inform experimental iteration, minimizing cost and maximizing data output.

Furthermore, these approaches will be essential in next-generation space-based facility development, where automation will be critical and machine learning and Al can be applied to improve manufacturing processes. The use of fully automated systems on future LEO-based platforms could remove the need for astronaut intervention altogether, which would eliminate the significant costs associated with developing platforms that are habitable for humans.

\section{Market Analysis}

Currently, rapid and significant advancements are being made in space infrastructure technologies that provide increased frequency and declining cost in accessing space. Development of such technologies is further supported by government procurements, particularly NASA's Next Space Technologies for Exploration Partnerships-2 Broad Agency Announcement and, more recently, NASA's Commercial Low-Earth Orbit Destinations announcement. As a result, we are seeing an increasing emergence of business plans from companies such as Axiom Space, Sierra Space, and others aiming to build free fliers and modular commercial space stations that will be available to a diverse set of users.

The development of such LEO-based platforms will build on the lessons learned from the ISS and will enable space-based R\&D and manufacturing capabilities that will provide an infrastructure for further evolution and maturation of various business cases and models over the coming years. To assess the potential financial impact that space-based biomanufacturing could have on the utilization of such space-based infrastructure and the evolution of a commercial LEO economy, we performed a preliminary market size analysis.

It is important to note that most of the biomanufacturing technologies discussed here, while potentially addressing disruptive opportunities, have very significant early-stage innovation and development risks, which could shift timelines materially. Hence, we have chosen to present our estimates below as a relatively conservative scenario. We fully understand that if a disruptive technology with positive economic potential is successfully developed, the productspecific growth rates that could be achieved could far exceed the compound annual growth rate (CAGR) metrics discussed below. Given all this, we estimate the market size of LEO-based biomanufacturing to reach $\$ 2.87 \mathrm{~B}$ in 2035 from $\$ 308 \mathrm{M}$ in 2026 at a CAGR of $25 \%$ for the period 2026-2035. We segmented the LEO biomanufacturing market into five primary subsegments: (1) cell and tissue tools and diagnostics, (2) cell and tissue therapy, (3) bioprinting, (4) cell therapy biomanufacturing, and (5) organoids.

For the preliminary market sizing analysis, market research reports were queried using the online University of Pittsburgh Library System and a general internet search. Market research 
databases included BCC Research, IBISWorld, and Transparency Market Research. Market data from these research reports were grouped by the five-primary market subsegments. The cell and tissue tools and diagnostics market was further subsegmented into seven markets: (1) cell line, (2) cell harvesting, (3) cell expansion, (4) cell and gene therapy tools and reagents, (5) cancer cell analysis, (6) cell viability assays, and (7) cell-based assays. The cell and tissue therapy market was subsegmented into four markets: (1) tissue engineering and regeneration, (2) stem cell and regenerative therapy, (3) induced pluripotent stem cells, and (4) cell therapy

\section{Biomanufacturing in Low Earth Orbit Market Subsegmentation Revenue Projection}

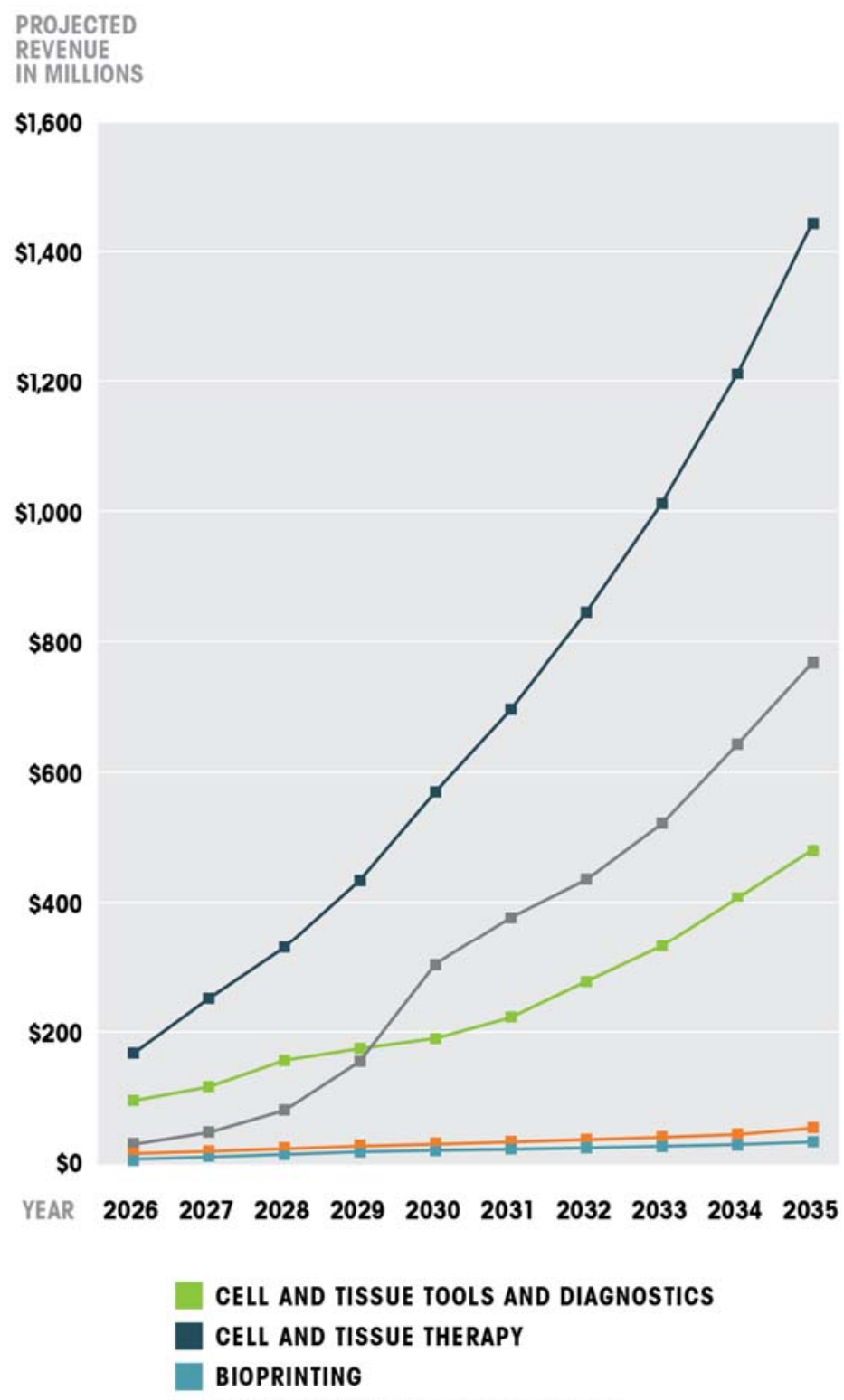

Figure 16: Biomanufacturing in low Earth orbit market subsegmentation revenue projection processing.

Because the market research reports had varying degrees of overlapping submarkets, the average of the market sizes for the tissue engineering and regeneration market and the stem cell and regenerative therapy market were used as the total cell and tissue therapy market size. The market reports provided a baseline terrestrial market size and projected market size, typically over a five-year span, and a CAGR. We used each CAGR to interpolate market sizes and to extrapolate through 2030.

These transformations provided yearly terrestrial market size forecasts from 2018 to 2030. LEO market sizes were forecasted starting in year 2026, providing for an approximate five-year market planning and development lead cycle (Figure 16). The cell and tissue tools and diagnostics LEO market was derived from a range of $0.05 \%$ to $0.10 \%$ of the associated terrestrial market estimates. An additional $10 \%$ per annum market growth over the terrestrial markets was applied through 2030 to account for relative advantages for those markets in LEO. From 2031 through the 2035 forecasted 
periods, the LEO market was forecasted to grow at 20\% per annum to reflect high growth markets after the five-year introductory period. The LEO cell and tissue therapy, bioprinting, and cell therapy biomanufacturing markets were forecasted using the same methods as above, except each was derived from $0.10 \%$ of the terrestrial markets.

The organoid LEO market was forecasted using a different method, based on this market being relatively new and anticipated to follow an exponential market growth curve. We modeled the organoid LEO after the CRISPR market because we believe these markets share similar exponential product and market adoption curves. Using published market forecast data for CRISPR, we calculated a market growth function of $y=369.34 e^{0.3564 x}$. Converting that function to the organoid LEO baseline market size of $\$ 6 \mathrm{M}$ in 2026, we calculated a market growth function of $y=4.2 e^{0.3564 x}$. This exponential growth formula was used to forecast the organoid LEO market size through 2030.

\section{Developing a Roadmap to Biomanufacturing in Space}

Biomanufacturing in space has the potential to enable scientific and technological advancements not achievable on the ground, leading to products that provide both economic value and benefits to life on Earth. R\&D supported by the ISS National Lab over the last decade in the areas of tissue engineering and regenerative medicine has laid the foundation for more complex studies and future investment in this area.

However, to develop a sustainable market in LEO, additional targeted R\&D is required to demonstrate the viability and economic value of space-based biomanufacturing. The ISS National Lab is uniquely positioned to enable this R\&D, and it is critical for current and potential future stakeholders to work collaboratively to identify the best opportunities to leverage the ISS to demonstrate the Earth-based benefits and return on investment provided by biomanufacturing in space.

The Biomanufacturing in Space Symposium was the first step in identifying and prioritizing the key opportunities to pursue. Symposium participants concluded that establishing a publicprivate consortium was the best way to advance these opportunities toward the development of a biomanufacturing marketplace in LEO. Continued public-sector funding is crucial to further de-risk space-based R\&D and facilitate investment and market growth. Private-sector involvement is also essential to guide the $R \& D$ to ensure it is focused on key marketplace needs.

A public-private consortium would serve to further prioritize and de-risk space-based biomanufacturing R\&D and translate results into commercial products for use in preclinical, clinical, and therapeutic applications on Earth. The consortium would combine traditional government-funded $R \& D$ with the requirements to raise and execute private-sector funding streams (commercial entities, venture capital, and startups) that could support not only 
individual space-based biomanufacturing projects but also the companies that will execute those projects on the ISS.

The next steps of a public-private consortium fall into three areas: (1) develop a structure and governance model to expedite the development and translation of biomanufacturing in LEO, (2) establish an integrated process outlining the role of the consortium from discovery to commercialization of a LEO-based product, and (3) recruit the right set of members for the consortium.

The structure and governance for the consortium should include an oversight board that sets priorities, provides resources, manages knowledge capture, and serves as a single point of contact for its membership and external key stakeholders. The board should include advisory committees of experts in three areas: industry/commercial, scientific/clinical, and LEO-based operations.

The consortium should follow a staged commercialization process going from a discovery/concept stage to taking a product to market. Each stage would have specific activities and critical milestones that must be met before a project could advance to the next stage. The advisory committees would be responsible for overseeing each stage and reporting back to the oversight board.

Recruiting the right set of expert members for the consortium is foundational to its success. Representation from five key groups will be needed on the oversight board and advisory committees: (1) commercial implementers (from pharmaceutical companies, contract development and manufacturing organizations, etc.); (2) technology developers from universities, institutes, and R\&D organizations; (3) technology enablers from companies and organizations with a focus on $\mathrm{Al}$, robotics, and automation; (4) launch and payload operations experts; and (5) public agencies (e.g., science, space, defense, and regulatory agencies). An ideal member would have knowledge and resources in multiple groups.

Commercialization for any new biomedical platform will take time, and the consortium could be defined by four phases that, taken together, span 10 years: (1) business foundation development; (2) research identification, prioritization, and development; (3) technology translation, approval, and application; and (4) technology manufacturing and commercialization. Based on discussions at the symposium, many of the key opportunities for biomanufacturing in space are in the second phase. For this reason, a productive consortium, as measured in commercial success, would require a minimum lifetime of five years (although ideally 10 years).

\section{Conclusions}

The last two decades have seen remarkable advances in tissue engineering and regenerative medicine as well as exponential advancement in space technologies that enable new 
opportunities to access and commercialize space for Earth benefit. We are now at an inflection point, where these areas can converge to capitalize on the unique advantages each confers. The Biomanufacturing in Space Symposium brought together thought leaders and subject matter experts to identify the most promising opportunities as well as the current gaps and pathways to realizing the full potential of utilizing the LEO environment for biomanufacturing.

Now is the time to leverage the ISS National Lab to conduct R\&D that demonstrates the economic value of space-based biomanufacturing and its benefits to life on Earth. This will enable the investment required to drive a robust biomanufacturing market in space. Investment in space-based biomanufacturing R\&D and a national infrastructure in LEO will be critical in maintaining U.S. leadership in this area as we transition to commercial LEO platforms of the future. The symposium was a first step toward this future, and we look forward to implementing a roadmap to successfully establish a sustainable market for biomanufacturing in space.

\section{Acknowledgements}

Funding to support the Biomanufacturing in Space Symposium and this report is from NSF/CMMI \#2028635 (MAG). We would like to thank Lucie Low (NCATS) for helping lead multiple sessions during the symposium and for providing a critical review of the manuscript. We would like to thank the following people*, not listed as authors, who gave presentations and participated as panelists during the symposium: Christopher P. Austin (NCATS), Anthony Atala (Wake Forest University), Eugene Boland (Techshot, Inc.), Christine M. Kretz (CASIS), Christian Maender (Axiom Space, Inc.), Erika Wagner (Blue Orgin), Phil McAllister (NASA), Twyman Clements (Space Tango, Inc.), Howie Choset (Carnegie Mellon University), Jun Auxup (Indie Bio), Edwin D. Trautman (Pfizer), William Proctor (Genentech), Andy Topping (Fujifilm Diosynth Biotechnologies), Teodoro Laino (IBM Research), Serena M. Auñón-Chancellor (NASA), Gordana Vunjak-Novakovic (Columbia University), Shirin Sohrabi (IBM Research), Alexander Godfrey (NCATS), Ricky Solorzano (Allevi), Binata Joddar (University of Texas, El Paso), Alysson R. Muotri (University of California, San Diego), Stefanie Countryman (BioServe Space Technologies), Christopher Hinojosa (Emulate, Inc.), Edward Kelly (University of Washington), Jana Stoudemire (Space Tango, Inc.), Abba C. Zubair (Mayo Clinic), Ilyas Singec (NCATS), Stephen Lin (The California Institute for Regenerative Medicine), Prashant Kumta (University of Pittsburgh), Chad Eckart (Johnson \& Johnson), Adam W. Feinberg (Carnegie Mellon University), Min Jae Song (NCATS), Bill McLamb (Space Tango, Inc.), Christopher J. Medberry (Johnson \& Johnson), Cynthia Martin-Brennan (Space Tango, Inc.), Hui Jenny Chen (3DHeals), Themasap Khan (Civilization Ventures), Tony Kulesa (Petri), Hector Martinez (Cellink AB), Mike Graffeo (FluidForm), Danilo Tagle (NCATS), Kendan Jones-Isaac (University of Washington), Peter H. U. Lee (The Ohio State University), Kristin M. Fabre (Translational Research Institute for Space Health), Steven M. Zehnder (NSF), Szczepan Baran (Novartis), Valentina Fossati (New York Stem Cell Foundation), Richard Egland (Arsenal Capital Partners), Lynn Harper (NASA), Rosemarie Hunziker (Connexion Life Science Consulting), Chris Scherzer (Techshot), Joseph Maggiore (University of Pittsburgh), Andrew Brown (University of Pittsburgh), Matthew P. Wagoner (Takeda Pharmaceuticals), Thomas Neumann (Nortis, Inc.), Russell Hannigan (Xplore), 
Elizabeth Blaber (Rensselaer Polytechnic Institute), Jennifer Manning (Fujifilm Diosynth Biotechnology), Chunhui Xu (Emory University), Eugenia Jones (Fujifilm Cellular Dynamics), Rudranarayan M. Mukherjee (Jet Propulsion Laboratory), Gary Fedder (Carnegie Mellon University), Gene Yeo (University of California, San Diego), Govind Kaigala (IBM Research), Sylvain Costes (NASA), George Muschler (CellX Technologies), Travis Block (StemBioSys, Inc.), Jonathan Somayajulu (Johnson \& Johnson), and Jordan Greco (LambaVision). We would thank Amy Elkavich (CASIS) for editorial support and Kathrine Wharton (McGowan Institute for Regenerative Medicine) for programmatic support. Rachel A. Clemens and Anjali Gupta are employees of Axiom Space, Inc. Nicole L. Wagner is an owner and employee of LambdaVision, Inc.

*Note: The affiliations listed here were their current affiliations at the time of the symposium.

\section{Author Contributions}

Conceptualization (MAG, GR, PC, WRW), Methodology (MAG, GR, PC, WRW, JB, EP), Validation (MAG, GR, PC, WRW, JB, EP), Formal Analysis (MAG, GR, PC, WRW, JB, EP, DPT), Resources (MAG, AS, RAC, OG, DLT, NLW, KAS, AJG, SM, AG, MK-J, DBM, D-HK, JFL, MSR, JH, LEW, JB, EP, $M R, D P T, G R, P C, A W S, W R W)$, Writing - Original Draft (MAG), Writing - Review \& Editing (MAG, AS, RAC, OG, DLT, NLW, KAS, AJG, SM, AG, MK-J, DBM, D-HK, JFL, MSR, JH, LEW, SE, JB, $E P, M R, D P T, G R, P C, A W S, W R W$ ), Visualization (MAG, EP, DPT, GR, AWS), Supervision (MAG, WRW), Project Administration (MAG, GR, PC, WRW), Funding Acquisition (MAG) 


\section{References}

2019 Report: U.S. Government Support of the Entrepreneurial Space Age.

https://sbir.nasa.gov/content/publications Accessed July 8, 2021

Acres, J.M., Youngapelian, M.J. \& Nadeau, J. The Influence of Spaceflight and Simulated Microgravity on Bacterial Motility and Chemotaxis. npj Microgravity 7, 7 (2021).

10.1038/s41526-021-00135-x

Ade, C. J., Broxterman, R. M., Charvat, J. M., \& Barstow, T. J. (2017). Incidence Rate of Cardiovascular Disease End Points in the National Aeronautics and Space Administration Astronaut Corps. Journal of the American Heart Association, 6(8), e005564.

10.1161/JAHA.117.005564

Ahari, H., Bedard, R., Bowes, C. et al. (1997) Effect of Microgravity on the Crystallization of A Self-assembling Layered Material. Nature 388, 857-860. 10.1038/42213

Ainslie, G. R., Davis, M., Ewart, L., Lieberman, L. A., Rowlands, D. J., Thorley, A. J., Yoder, G., \& Ryan, A. M. (2019). Microphysiological Lung Models to Evaluate the Safety of New Pharmaceutical Modalities: A Biopharmaceutical Perspective. Lab on a Chip, 19(19), 3152-3161. 10.1039/c9lc00492k

Akima, H., Kawakami, Y., Kubo, K., Sekiguchi, C., Ohshima, H., Miyamoto, A., \& Fukunaga, T. (2000). Effect of Short-Duration Spaceflight on Thigh and Leg Muscle Volume. Medicine and Science in Sports and Exercise, 32(10), 1743-1747. 10.1097/00005768-200010000-00013

Alliance for Regenerative Medicine. Q3 2019 Data Report, (2019) https://alliancerm.org/publication/q3-2019-data-report

An, J., Chua, C. K., \& Mironov, V. (2021). Application of Machine Learning in 3D Bioprinting: Focus on Development of Big Data and Digital Twin. International Journal of Bioprinting, 7(1), 342. 10.18063/ijb.v7i1.342

Baio, J., Martinez, A. F., Silva, I., Hoehn, C. V., Countryman, S., Bailey, L., Hasaniya, N., Pecaut, M. J., \& Kearns-Jonker, M. (2018). Cardiovascular Progenitor Cells Cultured Aboard the International Space Station Exhibit Altered Developmental and Functional Properties. NPJ Microgravity, 4, 13. 10.1038/s41526-018-0048-x

Bajpayee, A. G., \& Grodzinsky, A. J. (2017). Cartilage-targeting Drug Delivery: Can Electrostatic Interactions Help? Nature Reviews. Rheumatology, 13(3), 183-193. 10.1038/nrrheum.2016.210 
Baudy, A. R., Otieno, M. A., Hewitt, P., Gan, J., Roth, A., Keller, D., Sura, R., Van Vleet, T. R., \& Proctor, W. R. (2020). Liver Microphysiological Systems Development Guidelines for Safety Risk Assessment in the Pharmaceutical Industry. Lab on a Chip, 20(2), 215-225. 10.1039/c9lc00768g

Beaudart, C., Rizzoli, R., Bruyère, O., Reginster, J. Y., \& Biver, E. (2014). Sarcopenia: Burden and Challenges for Public Health. Archives of Public Health = Archives belges de sante publique, 72(1), 45. 10.1186/2049-3258-72-45

Benavides Damm, T., Walther, I., Wüest, S. L., Sekler, J., \& Egli, M. (2014). Cell Cultivation Under Different Gravitational Loads Using A Novel Random Positioning Incubator. Biotechnology and Bioengineering, 111(6), 1180-1190. 10.1002/bit.25179

Bergmann, O., Zdunek, S., Felker, A., Salehpour, M., Alkass, K., Bernard, S., Sjostrom, S. L., Szewczykowska, M., Jackowska, T., Dos Remedios, C., Malm, T., Andrä, M., Jashari, R., Nyengaard, J. R., Possnert, G., Jovinge, S., Druid, H., \& Frisén, J. (2015). Dynamics of Cell Generation and Turnover in the Human Heart. Cell, 161(7), 1566-1575.

10.1016/j.cell.2015.05.026

BioFabrication Facility (2021)

https://www.nasa.gov/mission_pages/station/research/experiments/explorer/Facility.html\#id= 7599 Accessed 8 July 2021.

Blaber, E. A., Finkelstein, H., Dvorochkin, N., Sato, K. Y., Yousuf, R., Burns, B. P., Globus, R. K., \& Almeida, E. A. (2015). Microgravity Reduces the Differentiation and Regenerative Potential of Embryonic Stem Cells. Stem Cells and Development, 24(22), 2605-2621.

$10.1089 /$ scd. 2015.0218

Bradbury, P., Wu, H., Choi, J. U., Rowan, A. E., Zhang, H., Poole, K., Lauko, J., \& Chou, J. (2020). Modeling the Impact of Microgravity at the Cellular Level: Implications for Human Disease. Frontiers in Cell and Developmental Biology, 8, 96. 10.3389/fcell.2020.00096

Cadena, S. M., Zhang, Y., Fang, J., Brachat, S., Kuss, P., Giorgetti, E., Stodieck, L. S., Kneissel, M., \& Glass, D. J. (2019). Skeletal Muscle in MuRF1 Null Mice Is Not Spared in Low-Gravity Conditions, Indicating Atrophy Proceeds by Unique Mechanisms in Space. Scientific Reports, 9(1), 9397. 10.1038/s41598-019-45821-9

Camberos, V., Baio, J., Bailey, L., Hasaniya, N., Lopez, L. V., \& Kearns-Jonker, M. (2019). Effects of Spaceflight and Simulated Microgravity on YAP1 Expression in Cardiovascular Progenitors: Implications for Cell-Based Repair. International journal of molecular sciences, 20(11), 2742. 10.3390/ijms20112742 
Camberos, V., Baio, J., Mandujano, A., Martinez, A. F., Bailey, L., Hasaniya, N., \& Kearns-Jonker, M. (2021). The Impact of Spaceflight and Microgravity on the Human Islet-1+ Cardiovascular Progenitor Cell Transcriptome. International Journal of Molecular Sciences, 22(7), 3577. 10.3390/ijms22073577

Carreras, P., González, I., Gallardo, M., Ortiz-Ruiz, A., Morales, M. L., Encinas, J., \& MartínezLópez, J. (2021). Long-Term Human Hematopoietic Stem Cell Culture in Microdroplets. Micromachines, 12(1), 90. 10.3390/mi12010090

Chakraborty, N., Waning, D. L., Gautam, A., Hoke, A., Sowe, B., Youssef, D., Butler, S., Savaglio, M., Childress, P. J., Kumar, R., Moyler, C., Dimitrov, G., Kacena, M. A., \& Hammamieh, R. (2020). Gene-Metabolite Network Linked to Inhibited Bioenergetics in Association with SpaceflightInduced Loss of Male Mouse Quadriceps Muscle. Journal of Bone and Mineral Research : The Official Journal of the American Society for Bone and Mineral Research, 35(10), 2049-2057. 10.1002/jbmr.4102

Chan, A., \& Huang, N. F. (2021). Engineering Cardiovascular Tissue Chips for Disease Modeling and Drug Screening Applications. Frontiers in Bioengineering and Biotechnology, 9, 673212. 10.3389/fbioe.2021.673212

Chiao, M. (2017) Microgravity: Space Worms with Two Heads. Nat Astron 1, 0183 10.1038/s41550-017-0183

Chivu-Economescu, M. and Rubach, M. (2017). Hematopoietic Stem Cells Therapies. Curr. Stem Cell Res. Ther., 12, 124-133. 10.2174/1574888X10666151026114241

Chlan L.L., Tofthagen C., Terzic A. (2019) The Regenerative Horizon: Opportunities for Nursing Research and Practice. J. Nurs. Scholarsh. 51, 651-660. 10.1111/jnu.12520.

Cohen-Karlik, E., Awida, Z., Bergman, A., Eshed, S., Nestor, O., Kadashev, M., Yosef, S. B., Saed, H., Mansour, Y., Globerson, A., Neumann, D., \& Gabet, Y. (2021). Quantification of Osteoclasts in Culture, Powered by Machine Learning. Frontiers in Cell and Developmental Biology, 9, 674710. 10.3389/fcell.2021.674710

Dag, O., Ahari, H., Coombs, N., Jiang, T., Aroca-Oullette, P. P., Petrov, S.; Sokolov, I., Verma, A., Vovk, G.;,Young, D., et al., (1997) Does Microgravity Influence Self-Assembly? Adv. Mater., 9, 1133-1149.

Daley, G. Q., Hyun, I., Apperley, J. F., Barker, R. A., Benvenisty, N., Bredenoord, A. L., Breuer, C. K., Caulfield, T., Cedars, M. I., Frey-Vasconcells, J., Heslop, H. E., Jin, Y., Lee, R. T., McCabe, C., Munsie, M., Murry, C. E., Piantadosi, S., Rao, M., Rooke, H. M., Sipp, D., ... Kimmelman, J. (2016). Setting Global Standards for Stem Cell Research and Clinical Translation: The 2016 ISSCR Guidelines. Stem Cell Reports, 6(6), 787-797. 10.1016/j.stemcr.2016.05.001 
da Silveira, W. A., Fazelinia, H., Rosenthal, S. B., Laiakis, E. C., Kim, M. S., Meydan, C., Kidane, Y., Rathi, K. S., Smith, S. M., Stear, B., Ying, Y., Zhang, Y., Foox, J., Zanello, S., Crucian, B., Wang, D., Nugent, A., Costa, H. A., Zwart, S. R., Schrepfer, S., ... Beheshti, A. (2020). Comprehensive Multiomics Analysis Reveals Mitochondrial Stress as a Central Biological Hub for Spaceflight Impact. Cell, 183(5), 1185-1201.e20. 10.1016/j.cell.2020.11.002

Delp, M. D., Charvat, J. M., Limoli, C. L., Globus, R. K., \& Ghosh, P. (2016). Apollo Lunar Astronauts Show Higher Cardiovascular Disease Mortality: Possible Deep Space Radiation Effects on the Vascular Endothelium. Scientific Reports, 6, 29901. 10.1038/srep29901

De Pieri, A., Rochev, Y., \& Zeugolis, D. I. (2021). Scaffold-free Cell-Based Tissue Engineering Therapies: Advances, Shortfalls and Forecast. NPJ Regenerative Medicine, 6(1), 18. 10.1038/s41536-021-00133-3

Dinkin S., (2019) How Low Can Launch Costs Go? The Space Review. https://thespacereview.com/article/3740/1

Djisalov, M., Knežić, T., Podunavac, I., Živojević, K., Radonic, V., Knežević, N. Ž., Bobrinetskiy, I., \& Gadjanski, I. (2021). Cultivating Multidisciplinarity: Manufacturing and Sensing Challenges in Cultured Meat Production. Biology, 10(3), 204. 10.3390/biology10030204

Donoghue, L., Nguyen, K. T., Graham, C., \& Sethu, P. (2021). Tissue Chips and Microphysiological Systems for Disease Modeling and Drug Testing. Micromachines, 12(2), 139. 10.3390/mi12020139

Ferranti, F., Del Bianco, M., \& Pacelli, C. (2020). Advantages and Limitations of Current Microgravity Platforms for Space Biology Research. Applied Sciences, 11(1), 68. 10.3390/app11010068

Fryar, C. D., Chen, T. C., \& Li, X. (2012). Prevalence of Uncontrolled Risk Factors for Cardiovascular Disease: United States, 1999-2010. NCHS data brief, (103), 1-8.

Fowler, S., Chen, W., Duignan, D. B., Gupta, A., Hariparsad, N., Kenny, J. R., Lai, W. G., Liras, J., Phillips, J. A., \& Gan, J. (2020). Microphysiological Systems for ADME-related Applications: Current Status and Recommendations for System Development and Characterization. Lab on a Chip, 20(3), 446-467. 10.1039/c9lc00857h

Gao, D., Morini, E., Salani, M., Krauson, A. J., Chekuri, A., Sharma, N., Ragavendran, A., Erdin, S., Logan, E. M., Li, W., Dakka, A., Narasimhan, J., Zhao, X., Naryshkin, N., Trotta, C. R., Effenberger, K. A., Woll, M. G., Gabbeta, V., Karp, G., Yu, Y., ... Slaugenhaupt, S. A. (2021). A Deep Learning Approach to Identify Gene Targets of A Therapeutic for Human Splicing Disorders. Nature Communications, 12(1), 3332. 10.1038/s41467-021-23663-2 
Garrett-Bakelman, F. E., Darshi, M., Green, S. J., Gur, R. C., Lin, L., Macias, B. R., McKenna, M. J., Meydan, C., Mishra, T., Nasrini, J., Piening, B. D., Rizzardi, L. F., Sharma, K., Siamwala, J. H., Taylor, L., Vitaterna, M. H., Afkarian, M., Afshinnekoo, E., Ahadi, S., Ambati, A., ... Turek, F. W. (2019). The NASA Twins Study: A Multidimensional Analysis of A Year-Long Human Spaceflight. Science (New York, N.Y.), 364(6436), eaau8650. 10.1126/science.aau8650

Gentile, P., Carmagnola, I., Nardo, T., \& Chiono, V. (2015). Layer-by-layer Assembly for Biomedical Applications in the Last Decade. Nanotechnology, 26(42), 422001. 10.1088/09574484/26/42/422001

Giulianotti, M. A., \& Low, L. A. (2019). Pharmaceutical Research Enabled Through Microgravity: Perspectives on the Use of the International Space Station U.S. National Laboratory. Pharmaceutical Research, 37(1), 1. 10.1007/s11095-019-2719-z

Grimm, D., Egli, M., Krüger, M., Riwaldt, S., Corydon, T. J., Kopp, S., Wehland, M., Wise, P., Infanger, M., Mann, V., \& Sundaresan, A. (2018). Tissue Engineering Under Microgravity Conditions-Use of Stem Cells and Specialized Cells. Stem Cells and Development, 27(12), 787804. 10.1089/scd.2017.0242

Grimm, D., Wehland, M., Pietsch, J., Aleshcheva, G., Wise, P., van Loon, J., Ulbrich, C., Magnusson, N. E., Infanger, M., \& Bauer, J. (2014). Growing Tissues in Real and Simulated Microgravity: New Methods for Tissue Engineering. Tissue Engineering. Part B, Reviews, 20(6), 555-566. 10.1089/ten.TEB.2013.0704

Guo, Q. R., Zhang, L. L., Liu, J. F., Li, Z., Li, J. J., Zhou, W. M., Wang, H., Li, J. Q., Liu, D. Y., Yu, X. Y., \& Zhang, J. Y. (2021). Multifunctional Microfluidic Chip for Cancer Diagnosis and Treatment. Nanotheranostics, 5(1), 73-89. 10.7150/ntno.49614

Gu, Z., Fu, J., Lin, H., \& He, Y. (2020). Development of 3D Bioprinting: From Printing Methods to Biomedical Applications. Asian Journal of Pharmaceutical Sciences, 15(5), 529-557.

10.1016/j.ajps.2019.11.003

Gungor-Ozkerim, P. S., Inci, I., , Zhang, Y. S., , Khademhosseini, A., , \& Dokmeci, M. R., (2018). Bioinks for 3D Bioprinting: An Overview. Biomaterials Science, 6(5), 915-946.

10.1039/c7bm00765e

Heinrich, M. A., Liu, W., Jimenez, A., Yang, J., Akpek, A., Liu, X., Pi, Q., Mu, X., Hu, N., Schiffelers, R. M., Prakash, J., Xie, J., \& Zhang, Y. S. (2019). 3D Bioprinting: From Benches to Translational Applications. Small (Weinheim an der Bergstrasse, Germany), 15(23), e1805510.

10.1002/smll.201805510 
Herranz, R., Anken, R., Boonstra, J., Braun, M., Christianen, P. C., de Geest, M., Hauslage, J., Hilbig, R., Hill, R. J., Lebert, M., Medina, F. J., Vagt, N., Ullrich, O., van Loon, J. J., \& Hemmersbach, R. (2013). Ground-based Facilities for Simulation of Microgravity: OrganismSpecific Recommendations for Their Use, and Recommended Terminology. Astrobiology, 13(1), 1-17. 10.1089/ast.2012.0876

Herrmann, M., Engelke, K., Ebert, R., Müller-Deubert, S., Rudert, M., Ziouti, F., Jundt, F., Felsenberg, D., \& Jakob, F. (2020). Interactions Between Muscle and Bone-Where Physics Meets Biology. Biomolecules, 10(3), 432. 10.3390/biom10030432

Huang, P., Russell, A. L., Lefavor, R., Durand, N. C., James, E., Harvey, L., Zhang, C., Countryman, S., Stodieck, L., \& Zubair, A. C. (2020). Feasibility, Potency, and Safety of Growing Human Mesenchymal Stem Cells in Space for Clinical Application. NPJ Microgravity, 6, 16.

10.1038/s41526-020-0106-z

Hunsberger, J., Harrysson, O., Shirwaiker, R., Starly, B., Wysk, R., Cohen, P., Allickson, J., Yoo, J., \& Atala, A. (2015). Manufacturing Road Map for Tissue Engineering and Regenerative Medicine Technologies. Stem Cells Translational Medicine, 4(2), 130-135. 10.5966/sctm.2014-0254

Hunsberger, J., Simon, C., Zylberberg, C., Ramamoorthy, P., Tubon, T., Bedi, R., Gielen, K., Hansen, C., Fischer, L., Johnson, J., Baraniak, P., Mahdavi, B., Pereira, T., Hadjisavas, M., Eaker, S., \& Miller, C. (2020). Improving Patient Outcomes with Regenerative Medicine: How the Regenerative Medicine Manufacturing Society Plans to Move the Needle Forward in Cell Manufacturing, Standards, 3D Bioprinting, Artificial Intelligence-Enabled Automation, Education, and Training. Stem Cells Translational Medicine, 9(7), 728-733. 10.1002/sctm.190389

Hysenaj, L., Little, S., Kulhanek, K., Gbenedio, O. M., Rodriguez, L., Shen, A., Lone, J. C., LupinJimenez, L. C., Bonser, L. R., Serwas, N. K., Bahl, K., Mick, E., Li, J. Z., Ding, V. W., Matsumoto, S., Maishan, M., Simoneau, C., Fragiadakis, G., Jablons, D. M., Langelier, C. R., ... Roose, J. P. (2021). SARS-CoV-2 Infection Studies in Lung Organoids Identify TSPAN8 as Novel Mediator. bioRxiv: The Preprint Server for Biology, 2021.06.01.446640. 10.1101/2021.06.01.446640

Imura, T., Otsuka, T., Kawahara, Y., \& Yuge, L. (2019). "Microgravity" As A Unique and Useful Stem Cell Culture Environment for Cell-Based Therapy. Regenerative Therapy, 12, 2-5. 10.1016/j.reth.2019.03.001

Implementation Partners (2021) https://www.issnationallab.org/implementation-partners Accessed 8 July 2021.

International Space Station Benefits for Humanity, 3rd Edition (2019). Available from https://www.nasa.gov/mission_pages/station/research/news/b4h-3rd-ed-book. Accessed 7 July 2021 
International Stem Cell Initiative (2018). Assessment of Established Techniques to Determine Developmental and Malignant Potential of Human Pluripotent Stem Cells. Nature communications, 9(1), 1925. 10.1038/s41467-018-04011-3

Janssen, I., Shepard, D. S., Katzmarzyk, P. T., \& Roubenoff, R. (2004). The Healthcare Costs of Sarcopenia in the United States. Journal of the American Geriatrics Society, 52(1), 80-85. 10.1111/j.1532-5415.2004.52014.x

Jha, R., Wu, Q., Singh, M., Preininger, M. K., Han, P., Ding, G., Cho, H. C., Jo, H., Maher, K. O., Wagner, M. B., \& Xu, C. (2016). Simulated Microgravity and 3D Culture Enhance Induction, Viability, Proliferation and Differentiation of Cardiac Progenitors from Human Pluripotent Stem Cells. Scientific Reports, 6, 30956. 10.1038/srep30956

Jones, H. W. (2020) The Recent Large Reduction in Space Launch Cost. International Conference on Environmental Systems. https://ntrs.nasa.gov/citations/20200001093

Jovic, T. H., Combellack, E. J., Jessop, Z. M., \& Whitaker, I. S. (2020). 3D Bioprinting and the Future of Surgery. Frontiers in Surgery, 7, 609836. 10.3389/fsurg.2020.609836

Kimmel, J. C., Yi, N., Roy, M., Hendrickson, D. G., and Kelley, D. R. (2021). Differentiation Reveals Latent Features of Aging and an Energy Barrier in Murine Myogenesis. Cell Reports, 35(4), 109046. 10.1016/j.celrep.2021.109046

Krüger, M., Melnik, D., Kopp, S., Buken, C., Sahana, J., Bauer, J., Wehland, M., Hemmersbach, R., Corydon, T. J., Infanger, M., \& Grimm, D. (2019). Fighting Thyroid Cancer with Microgravity Research. International Journal of Molecular Sciences, 20(10), 2553. 10.3390/ijms20102553

Lawler, J. M., Hord, J. M., Ryan, P., Holly, D., Janini Gomes, M., Rodriguez, D., Guzzoni, V., Garcia-Villatoro, E., Green, C., Lee, Y., Little, S., Garcia, M., Hill, L., Brooks, M. C., Lawler, M. S., Keys, N., Mohajeri, A., \& Kamal, K. Y. (2021). Nox2 Inhibition Regulates Stress Response and Mitigates Skeletal Muscle Fiber Atrophy during Simulated Microgravity. International Journal of Molecular Sciences, 22(6), 3252. 10.3390/ijms22063252

Lee, H., Kim, W., Lee, J., Park, K. S., Yoo, J. J., Atala, A., Kim, G. H., \& Lee, S. J. (2021). Self-aligned Myofibers in 3D Bioprinted Extracellular Matrix-Based Construct Accelerate Skeletal Muscle Function Restoration. Applied Physics Reviews, 8(2), 021405. 10.1063/5.0039639

Lee, J., Oh, S. J., An, S. H., Kim, W. D., \& Kim, S. H. (2020). Machine Learning-based Design Strategy for 3D Printable Bioink: Elastic Modulus and Yield Stress Determine Printability. Biofabrication, 12(3), 035018. 10.1088/1758-5090/ab8707

Lee, S., Feiveson, A. H., Stein, S., Stenger, M. B., \& Platts, S. H. (2015). Orthostatic Intolerance After ISS and Space Shuttle Missions. Aerospace Medicine and Human Performance, 86(12 Suppl), A54-A67. 10.3357/AMHP.EC08.2015 
Li, Z., Zhang, Z., Ren, Y., Wang, Y., Fang, J., Yue, H., Ma, S., \& Guan, F. (2021). Aging and AgeRelated Diseases: From Mechanisms to Therapeutic Strategies. Biogerontology, 22(2), 165-187. 10.1007/s10522-021-09910-5

Low, L. A., \& Giulianotti, M. A. (2019). Tissue Chips in Space: Modeling Human Diseases in Microgravity. Pharmaceutical Research, 37(1), 8. 10.1007/s11095-019-2742-0

Low, L. A., Mummery, C., Berridge, B. R., Austin, C. P., \& Tagle, D. A. (2021). Organs-on-chips: Into the Next Decade. Nature Reviews. Drug Discovery, 20(5), 345-361. 10.1038/s41573-0200079-3

Luxton, J. J., \& Bailey, S. M. (2021). Twins, Telomeres, and Aging-in Space! Plastic and Reconstructive Surgery, 147(1S-2), 7S-14S. 10.1097/PRS.0000000000007616

Ma, L., Wu, Y., Li, Y., Aazmi, A., Zhou, H., Zhang, B., \& Yang, H. (2020). Current Advances on 3DBioprinted Liver Tissue Models. Advanced Healthcare Materials, 9(24), e2001517. 10.1002/adhm.202001517

Mandrycky, C., Wang, Z., Kim, K., \& Kim, D. H. (2016). 3D Bioprinting for Engineering Complex Tissues. Biotechnology Advances, 34(4), 422-434. 10.1016/j.biotechadv.2015.12.011

Master S., Koshy N., Mansour R., Shi R.H. (2019) Effect of Stem Cell Transplant on Survival in Adult Patients with Acute Lymphoblastic Leukemia: NCDB Analysis. Anticancer Res, 39, 18991906. 10.21873/anticanres.13298.

McPherson, A., \& DeLucas, L. J. (2015). Microgravity Protein Crystallization. NPJ Microgravity, 1, 15010. 10.1038/npjmgrav.2015.10

Meck, J. V., Reyes, C. J., Perez, S. A., Goldberger, A. L., \& Ziegler, M. G. (2001). Marked Exacerbation of Orthostatic Intolerance After Long- vs. Short-Duration Spaceflight in Veteran Astronauts. Psychosomatic Medicine, 63(6), 865-873. 10.1097/00006842-200111000-00003

Mitchell, J. M., James Nemesh, Sulagna Ghosh, Robert E. Handsaker, Curtis J. Mello, Daniel Meyer, Kavya Raghunathan, Heather de Rivera, Matt Tegtmeyer, Derek Hawes, Anna Neumann, Ralda Nehme, Kevin Eggan, Steven A. McCarroll (2020) Mapping Genetic Effects on Cellular Phenotypes with "Cell Villages." bioRxiv, 2020.06.29.174383; doi: https://doi.org/10.1101/2020.06.29.174383

Moldovan N. I. (2018). Progress in Scaffold-free Bioprinting for Cardiovascular Medicine. Journal of Cellular and Molecular Medicine, 22(6), 2964-2969. 10.1111/jcmm.13598 
Mota, S. M., Rogers, R. E., Haskell, A. W., McNeill, E. P., Kaunas, R., Gregory, C. A., Giger, M. L., \& Maitland, K. C. (2021). Automated Mesenchymal Stem Cell Segmentation and Machine Learning-based Phenotype Classification Using Morphometric and Textural Analysis. Journal of Medical Imaging (Bellingham, Wash.), 8(1), 014503. 10.1117/1.JMI.8.1.014503

Tissue Chips in Space (2021). https://ncats.nih.gov/tissuechip/projects/space Accessed 8 July 2021.

Ntege, E. H., Sunami, H., \& Shimizu, Y. (2020). Advances in Regenerative Therapy: A Review of the Literature and Future Directions. Regenerative Therapy, 14, 136-153.

10.1016/j.reth.2020.01.004

Ortman, J., Velkoff, V., H., H., (2014) An Aging Nation: The Older Population in the United States. Current Population Reports. U.S. Department of Commerce Economics and Statistics Administration 2014.

Otsuka, T., Imura, T., Nakagawa, K., Shrestha, L., Takahashi, S., Kawahara, Y., Sueda, T., Kurisu, K., \& Yuge, L. (2018). Simulated Microgravity Culture Enhances the Neuroprotective Effects of Human Cranial Bone-Derived Mesenchymal Stem Cells in Traumatic Brain Injury. Stem Cells andDevelopment, 27(18), 1287-1297. 10.1089/scd.2017.0299

Patel S. (2020). The Effects of Microgravity and Space Radiation on Cardiovascular Health: From Low-Earth Orbit and Beyond. International journal of cardiology. Heart \& Vasculature, 30, 100595. 10.1016/j.ijcha.2020.100595

Pedde, R. D., Mirani, B., Navaei, A., Styan, T., Wong, S., Mehrali, M., Thakur, A., Mohtaram, N. K., Bayati, A., Dolatshahi-Pirouz, A., Nikkhah, M., Willerth, S. M., \& Akbari, M. (2017). Emerging Biofabrication Strategies for Engineering Complex Tissue Constructs. Advanced materials (Deerfield Beach, Fla.), 29(19), 10.1002/adma.201606061. 10.1002/adma.201606061

Perez-Bermejo, J. A., Kang, S., Rockwood, S. J., Simoneau, C. R., Joy, D. A., Silva, A. C., Ramadoss, G. N., Flanigan, W. R., Fozouni, P., Li, H., Chen, P. Y., Nakamura, K., Whitman, J. D., Hanson, P. J., McManus, B. M., Ott, M., Conklin, B. R., \& McDevitt, T. C. (2021). SARS-CoV-2 Infection of Human iPSC-derived Cardiac Cells Reflects Cytopathic Features in Hearts of Patients with COVID-19. Science Translational Medicine, 13(590), eabf7872. 10.1126/scitranslmed.abf7872

Peterson, N. C., Mahalingaiah, P. K., Fullerton, A., \& Di Piazza, M. (2020). Application of Microphysiological Systems in Biopharmaceutical Research and Development. Lab on a Chip, 20(4), 697-708. 10.1039/c9lc00962k

Phillips, J. A., Grandhi, T., Davis, M., Gautier, J. C., Hariparsad, N., Keller, D., Sura, R., \& Van Vleet, T. R. (2020). A Pharmaceutical Industry Perspective on Microphysiological Kidney Systems for Evaluation of Safety for New Therapies. Lab on a Chip, 20(3), 468-476. 10.1039/c9lc00925f 
Phua, Q. H., Han, H. A., and Soh, B. S. (2021). Translational Stem Cell Therapy: Vascularized Skin Grafts in Skin Repair and Regeneration. Journal of Translational Medicine, 19(1), 83. h10.1186/s12967-021-02752-2

Prasad, B., Richter, P., Vadakedath, N., Mancinelli, R., Krüger, M., Strauch, S. M., Grimm, D., Darriet, P., Chapel, J. P., Cohen, J., \& Lebert, M. (2020). Exploration of Space to Achieve Scientific Breakthroughs. Biotechnology Advances, 43, 107572.

10.1016/j.biotechadv.2020.107572

Pubmed Stem Cell 2021

https://pubmed.ncbi.nlm.nih.gov/?term=\%22stem+cell\%22\&filter=years.1992-

2022\&timeline=expanded\&sort=date Accessed 9 July 2021.

Reichert, P., Prosise, W., Fischmann, T. O., Scapin, G., Narasimhan, C., Spinale, A., Polniak, R., Yang, X., Walsh, E., Patel, D., Benjamin, W., Welch, J., Simmons, D., \& Strickland, C. (2019). Pembrolizumab Microgravity Crystallization Experimentation. NPJ microgravity, 5, 28.

10.1038/s41526-019-0090-3

Research and Markets (2021). Global Mesenchymal Stem Cells (MSC) Market Report 20212027: Market Competitors are Exploring Commercialization Strategies for MSC-Derived Extracellular Vesicles (EVs) and Exosomes. https://www.prnewswire.com/news-releases/globalmesenchymal-stem-cells-msc-market-report-2021-2027-market-competitors-areexploringcommercialization-strategies-for-msc-derived-extracellular-vesicles-evs-and-exosomes301228171.html Accessed 8 July 2021.

Richardson, J. J., Björnmalm, M., \& Caruso, F. (2015). Multilayer Assembly. Technology-driven Layer-by-layer Assembly of Nanofilms. Science (New York, N.Y.), 348(6233), aaa2491. 10.1126/science.aaa2491

Rodríguez-Fuentes, D. E., Fernández-Garza, L. E., Samia-Meza, J. A., Barrera-Barrera, S. A., Caplan, A. I., \& Barrera-Saldaña, H. A. (2021). Mesenchymal Stem Cells Current Clinical Applications: A Systematic Review. Archives of Medical Research, 52(1), 93-101. 10.1016/j.arcmed.2020.08.006

Sayed, N., Liu, C., \& Wu, J. C. (2016). Translation of Human-induced Pluripotent Stem Cells: From Clinical Trial in a Dish to Precision Medicine. Journal of the American College of Cardiology, 67(18), 2161-2176. 10.1016/j.jacc.2016.01.083

Semple, C., Riveros, D., Sung, D. M., Nagy, J. A., Rutkove, S. B., \& Mortreux, M. (2020). Using Electrical Impedance Myography as a Biomarker of Muscle Deconditioning in Rats Exposed to Micro- and Partial-Gravity Analogs. Frontiers in Physiology, 11, 557796.

10.3389/fphys.2020.557796 
Sharma, A., Garcia, G., Jr, Wang, Y., Plummer, J. T., Morizono, K., Arumugaswami, V., \& Svendsen, C. N. (2020). Human iPSC-Derived Cardiomyocytes Are Susceptible to SARS-CoV-2 Infection. Cell Reports. Medicine, 1(4), 100052. 10.1016/j.xcrm.2020.100052

Sharma, A., Sances, S., Workman, M. J., \& Svendsen, C. N. (2020). Multi-lineage Human iPSCDerived Platforms for Disease Modeling and Drug Discovery. Cell Stem Cell, 26(3), 309-329. 10.1016/j.stem.2020.02.011

Shelhamer, M., Bloomberg, J., LeBlanc, A., Prisk, G. K., Sibonga, J., Smith, S. M., Zwart, S. R., \& Norsk, P. (2020). Selected Discoveries from Human Research in Space That Are Relevant to Human Health on Earth. NPJ Microgravity, 6, 5. 10.1038/s41526-020-0095-y

Si, L., Bai, H., Rodas, M., Cao, W., Oh, C. Y., Jiang, A., Moller, R., Hoagland, D., Oishi, K., Horiuchi, S., Uhl, S., Blanco-Melo, D., Albrecht, R. A., Liu, W. C., Jordan, T., Nilsson-Payant, B. E., Golynker, I., Frere, J., Logue, J., Haupt, R., ... Ingber, D. E. (2021). A Human-airway-on-a-chip for the Rapid Identification of Candidate Antiviral Therapeutics and Prophylactics. Nature Biomedical Engineering, 10.1038/s41551-021-00718-9. Advance online publication. 10.1038/s41551-02100718-9

Simões, M. F., \& Antunes, A. (2021). Microbial Pathogenicity in Space. Pathogens (Basel, Switzerland), 10(4), 450. https://doi.org/10.3390/pathogens10040450

Smith J. K. (2020). Osteoclasts and Microgravity. Life (Basel, Switzerland), 10(9), 207. 10.3390/life10090207

Smith, R. C., Cramer, M. S., Mitchell, P. J., Lucchesi, J., Ortega, A. M., Livingston, E. W., Ballard, D., Zhang, L., Hanson, J., Barton, K., Berens, S., Credille, K. M., Bateman, T. A., Ferguson, V. L., Ma, Y. L., \& Stodieck, L. S. (2020). Inhibition of Myostatin Prevents Microgravity-induced Loss of Skeletal Muscle Mass and Strength. PloS One, 15(4), e0230818. 10.1371/journal.pone.0230818

Stern, J. H., Tian, Y., Funderburgh, J., Pellegrini, G., Zhang, K., Goldberg, J. L., Ali, R. R., Young, M., Xie, Y., \& Temple, S. (2018). Regenerating Eye Tissues to Preserve and Restore Vision. Cell Stem Cell, 22(6), 834-849. 10.1016/j.stem.2018.05.013

Tchkonia, T., Palmer, A. K., \& Kirkland, J. L. (2021). New Horizons: Novel Approaches to Enhance Healthspan Through Targeting Cellular Senescence and Related Aging Mechanisms. The Journal of Clinical Endocrinology and Metabolism, 106(3), e1481-e1487. 10.1210/clinem/dgaa728

Thomas, A. C., Hubbard-Turner, T., Wikstrom, E. A., \& Palmieri-Smith, R. M. (2017). Epidemiology of Posttraumatic Osteoarthritis. Journal of Athletic Training, 52(6), 491-496. 10.4085/1062-6050-51.5.08 
Travers, J. G., Kamal, F. A., Robbins, J., Yutzey, K. E., \& Blaxall, B. C. (2016). Cardiac Fibrosis: The Fibroblast Awakens. Circulation Research, 118(6), 1021-1040.

10.1161/CIRCRESAHA.115.306565

Topal, U., \& Zamur, C. (2021). Microgravity, Stem Cells, and Cancer: A New Hope for Cancer Treatment. Stem Cells International, 2021, 5566872. 10.1155/2021/5566872

Valuates Reports (2021). Organ-On-Chip (OOC) Market Size is USD 303.6 Million by 2026 at CAGR 39.9\% | Valuates Reports https://www.prnewswire.com/in/news-releases/organ-onchip-ooc-market-size-is-usd-303-6-million-by-2026-at-cagr-39-9-valuates-reports856995001.html Accessed 8 July 2021.

Varkey, M., Visscher, D. O., van Zuijlen, P., Atala, A., \& Yoo, J. J. (2019). Skin Bioprinting: The Future of Burn Wound Reconstruction? Burns \& Trauma, 7, 4. 10.1186/s41038-019-0142-7

Vernikos, J., \& Schneider, V. S. (2010). Space, Gravity and the Physiology of Aging: Parallel or Convergent Disciplines? A Mini-Review. Gerontology, 56(2), 157-166. 10.1159/000252852

Vieira, C. P., McCarrel, T. M., \& Grant, M. B. (2021). Novel Methods to Mobilize, Isolate, and Expand Mesenchymal Stem Cells. International Journal of Molecular Sciences, 22(11), 5728. 10.3390/ijms22115728

Virani, S. S., Alonso, A., Benjamin, E. J., Bittencourt, M. S., Callaway, C. W., Carson, A. P., Chamberlain, A. M., Chang, A. R., Cheng, S., Delling, F. N., Djousse, L., Elkind, M., Ferguson, J. F., Fornage, M., Khan, S. S., Kissela, B. M., Knutson, K. L., Kwan, T. W., Lackland, D. T., Lewis, T. T., ... American Heart Association Council on Epidemiology and Prevention Statistics Committee and Stroke Statistics Subcommittee (2020). Heart Disease and Stroke Statistics-2020 Update: A Report from the American Heart Association. Circulation, 141(9), e139-e596.

10.1161/CIR.0000000000000757

Wahlster, L., \& Daley, G. Q. (2016). Progress Towards Generation of Human Haematopoietic Stem Cells. Nature Cell Niology, 18(11), 1111-1117. 10.1038/ncb3419

Walasek, M. A., van Os, R., \& de Haan, G. (2012). Hematopoietic Stem Cell Expansion:

Challenges and Opportunities. Annals of the New York Academy of Sciences, 1266, 138-150. 10.1111/j.1749-6632.2012.06549.x

Wazir, U., Tayeh, S., Orakzai, M., Martin, T. A., Jiang, W. G., \& Mokbel, K. (2020). Stratification Using hTERT and Stem Cell Markers Confers a Good Prognosis in Invasive Breast Cancer. Cancer Genomics \& Proteomics, 17(2), 169-174. 10.21873/cgp.20177

Wertz, James R., and Larson, Wiley (1996) Reducing Space Mission Cost. J.R. Wertz and W Larson, ed (Netherlands: Springer Netherlands), pp. 115-117. 
Wnorowski, A., Sharma, A., Chen, H., Wu, H., Shao, N. Y., Sayed, N., Liu, C., Countryman, S., Stodieck, L. S., Rubins, K. H., Wu, S. M., Lee, P., \& Wu, J. C. (2019). Effects of Spaceflight on Human Induced Pluripotent Stem Cell-Derived Cardiomyocyte Structure and Function. Stem Cell Reports, 13(6), 960-969. 10.1016/j.stemcr.2019.10.006

Wuest, S. L., Richard, S., Kopp, S., Grimm, D., \& Egli, M. (2015). Simulated Microgravity: Critical Review on the Use of Random Positioning Machines for Mammalian Cell Culture. BioMed Research International, 2015, 971474. 10.1155/2015/971474

Yi, H. G., Kim, H., Kwon, J., Choi, Y. J., Jang, J., \& Cho, D. W. (2021). Application of 3D bioprinting in the prevention and the therapy for human diseases. Signal Transduction and Targeted Therapy, 6(1), 177. 10.1038/s41392-021-00566-8

Yim, E. K., \& Sheetz, M. P. (2012). Force-dependent Cell Signaling in Stem Cell Differentiation. Stem Cell Research \& Therapy, 3(5), 41. 10.1186/scrt132

Yu, Q., Kilik, U., Holloway, E. M., Tsai, Y. H., Harmel, C., Wu, A., Wu, J. H., Czerwinski, M., Childs, C. J., He, Z., Capeling, M. M., Huang, S., Glass, I. A., Higgins, P., Treutlein, B., Spence, J. R., \& Camp, J. G. (2021). Charting Human Development Using A Multi-Endodermal Organ Atlas and Organoid Models. Cell, 184(12), 3281-3298.e22. 10.1016/j.cell.2021.04.028

Yuge, L., Kajiume, T., Tahara, H., Kawahara, Y., Umeda, C., Yoshimoto, R., Wu, S. L., Yamaoka, K., Asashima, M., Kataoka, K., \& Ide, T. (2006). Microgravity Potentiates Stem Cell Proliferation While Sustaining the Capability of Differentiation. Stem Cells and Development, 15(6), 921-929. $\backslash 10.1089 /$ scd.2006.15.921

Yusuf, E. (2016) Pharmacologic and Non-Pharmacologic Treatment of Osteoarthritis. Curr Treat Options in Rheum 2, 111-125 10.1007/s40674-016-0042-y

Zea, L., McLean, R., Rook, T. A., Angle, G., Carter, D. L., Delegard, A., Denvir, A., Gerlach, R., Gorti, S., Mcllwaine, D., Nur, M., Peyton, B. M., Stewart, P. S., Sturman, P., \& Velez Justiniano, Y. A. (2020). Potential Biofilm Control Strategies for Extended Spaceflight Missions. Biofilm, 2, 100026. 10.1016/j.bioflm.2020.100026

Zhang, S., \& Wang, H. (2019). Current Progress in 3D Bioprinting of Tissue Analogs. SLAS Technology, 24(1), 70-78. 10.1177/2472630318799971

Zhang, Y., Ravikumar, M., Ling, L., Nurcombe, V., \& Cool, S. M. (2021). Age-related Changes in the Inflammatory Status of Human Mesenchymal Stem Cells: Implications for Cell Therapy. Stem Cell Reports, 16(4), 694-707. 10.1016/j.stemcr.2021.01.021 